\title{
Continuous-Time Mean Field Games with Finite State Space and Common Noise
}

\author{
Christoph Belak ${ }^{1}$ - Daniel Hoffmann ${ }^{2}$. Frank T. Seifried ${ }^{2}$
}

Accepted: 26 December 2020 / Published online: 7 February 2021

(c) The Author(s) 2021

\begin{abstract}
We formulate and analyze a mathematical framework for continuous-time mean field games with finitely many states and common noise, including a rigorous probabilistic construction of the state process and existence and uniqueness results for the resulting equilibrium system. The key insight is that we can circumvent the master equation and reduce the mean field equilibrium to a system of forward-backward systems of (random) ordinary differential equations by conditioning on common noise events. In the absence of common noise, our setup reduces to that of Gomes, Mohr and Souza (Appl Math Optim 68(1): 99-143, 2013) and Cecchin and Fischer (Appl Math Optim 81(2):253-300, 2020).
\end{abstract}

Keywords Mean field games $\cdot$ Common noise $\cdot$ Markov chains $\cdot$ Regime shifts

Mathematics Subject Classification 60J27 · 93E20 · 91A15

\section{Introduction}

Since the seminal contributions of Lasry and Lions [44] and Huang, Malhamé and Caines [39], mean field games have become an active field of mathematical research with a wide range of applications, including economics [13,16,27,33,41,50], sociology

$\bowtie$ Christoph Belak

belak@math.tu-berlin.de

Daniel Hoffmann

d.hoffmann@uni-trier.de

Frank T. Seifried

seifried@uni-trier.de

1 Faculty II - Mathematics and Natural Sciences, Institute of Mathematics, Technische Universität Berlin, Straße des 17. Juni 136, 10623 Berlin, Germany

2 Department IV - Mathematics, University of Trier, Universitätsring 19, 54296 Trier, Germany 
[35], finance [17,45], epidemiology [23,26,46] and computer science [40]; see also the overview article [29] and the monograph [9].

Mean field games constitute a class of dynamic, multi-player stochastic differential games with identical agents. The key characteristic of the mean field approach is that (i) the payoff and state dynamics of each agent depend on other agents' decisions only through an aggregate statistic (typically, the aggregate distribution of states); and (ii) no individual agent's actions can change the aggregate outcome. Thus, in solving an individual agent's optimization problem, the feedback effect of his own actions on the aggregate outcome can be discarded, breaking the notorious vicious circle ("the optimal strategy depends on the aggregate outcome, which depends on the strategy, which depends ..."). This significantly facilitates the identification of rational expectations equlibria. A standard assumption that further simplifies the analysis is that randomness is idiosyncratic (equivalently, there is no common noise), i.e. that the random variables appearing in one agent's optimization are independent of those in any other's. As a result, all randomness is "averaged out" in the aggregation of individual decisions, and the equilibrium dynamics of the aggregate distribution are deterministic.

In the literature, mean field games are most often studied in settings with a continuous state space and deterministic or diffusive dynamics, i.e. stochastic differential equations (SDEs) driven by Brownian motion. The corresponding dynamic programming equations thus become parabolic partial differential equations, and the aggregate dynamics are represented by a flow of Borel probability measures; see, e.g., the monographs [4] and [9] and the references therein. Formally, the mean field game is typically formulated in terms of a controlled McKean-Vlasov SDE, where the coefficients depend on the current state and control and the distribution of the solution; intuitively, these McKean-Vlasov dynamics codify the dynamics that pertain to a representative agent. The mathematical link to $N$-player games is subsequently made through suitable propagation of chaos results in the mean field limit $N \rightarrow \infty$; see, e.g., $[14,25,28,42,43]$. In this context, the analysis of McKean-Vlasov SDEs has also seen significant progress recently; see, e.g., $[6,8,19,48]$. In the presence of common noise, i.e. sources of risk that affect all agents and do not average out in the mean field limit, the mathematical analysis becomes even more involved as the dynamics of the aggregate distribution become stochastic, leading to conditional McKean-Vlasov dynamics; see, e.g., [1,12,21,51]. We refer to [10] for background and further references on continuous-state mean field games with common noise.

There is also a strand of literature on mean field games with finite state spaces, including $[2,15,18,24,30,31,34,49]$ as well as [9, \$7.2]. In a recent article, [22] provide an extension of [31] to mean field interactions that occur not only through the agents' states, but also through their controls. To the best of our knowledge, however, to date there has been no extension of these results to settings that include common noise. In the context of finite-state mean field games, we are only aware of two contributions that include common stochasticity (both via the master equation and with a different focus/setting than this paper): [5] analyze the master equation for finite-state mean field games with common noise, and [3] include a common continuous-time Gaussian noise in the aggregate distribution dynamics. 
In this article, we set up a mathematical framework for finite-state mean field games with common noise. ${ }^{1}$ Our setup extends that of [31] and [15] by common noise events at fixed points in time. We provide a rigorous formulation of the underlying stochastic dynamics, and we establish a verification theorem for the optimal strategy and an aggregation theorem to determine the resulting aggregate distribution. This leads to a characterization of the mean field equilibrium in terms of a system of (random) forward-backward differential equations. The key insight is that, after conditioning on common noise configurations, we obtain classical piecewise dynamics subject to jump conditions at common noise times.

The remainder of this article is organized as follows: In Sect. 2 we set up the mathematical model, provide a probabilistic construction of the state dynamics, and formulate the agent's optimization problem. In Sect. 3 we state the dynamic programming equation and establish a verification theorem for the agent's optimization, given an ex ante aggregate distribution (Theorem 6). Section 4 provides the dynamics of the ex post distribution (Theorem 9) and, on that basis, a system of random forward-backward ODEs for the mean field equilibrium (Definition 10) as well as corresponding existence and uniqueness results (Theorems 13 and 16 ). In Sect. 5 we showcase our results in two benchmark applications: agricultural production and infection control. The Appendix provides the proofs of Theorems 13 and 16.

\section{Mean Field Model}

We first provide an informal description of the individual agents' state dynamics, optimization problem, and the resulting mean field equilibrium. The agent's state process $X=\left\{X_{t}\right\}$ takes values in the finite set $\mathbb{S}$. Between common noise events, transitions from state $i$ to state $j$ occur with intensity $Q^{i j}\left(t, W_{t}, M_{t}, v_{t}\right)$, where $W_{t}$ represents the common noise events that have occurred up to time $t ; M_{t}$ the time$t$ aggregate distribution of agents; and $v_{t}$ the agent's control. In addition, upon the realization of a common noise event $W_{k}$ at time $T_{k}$, the state jumps from $X_{T_{k}-}$ to $X_{T_{k}}=J^{X_{T_{k}-}}\left(T_{k}, W_{T_{k}}, M_{T_{k}-}\right)$. With this, the agent aims to maximize

$$
\mathbb{E}^{v}\left[\int_{0}^{T} \psi^{X_{t}}\left(t, W_{t}, M_{t}, v_{t}\right) \mathrm{d} t+\Psi^{X_{T}}\left(W_{T}, M_{T}\right)\right]
$$

where $\psi$ and $\Psi$ are suitable reward functions and the aggregate distribution process $M=\left\{M_{t}\right\}$ is given by

$$
M_{t} \triangleq \mu\left(t, W_{t}\right) \text { for } t \in[0, T]
$$

Here $\mu$ represents the aggregate distribution of states as a function of the common noise factors. We obtain a rational expectations equilibrium by determining $\mu$ such that

\footnotetext{
${ }^{1}$ We wish to point out that our focus is not on the mean field limit of multi-player games; rather, we directly investigate the mean field equilibrium via the corresponding McKean-Vlasov dynamics (see also Remark 7 and [11] in that context).
} 
the representative agent's ex ante expectations equal the ex post aggregate distribution resulting from all agents' optimal decisions, i.e.

$$
\mathbb{P}^{\widehat{v}}\left(X_{t} \in \cdot \mid W_{t}\right)=\widehat{\mu}\left(t, W_{t}\right) \text { for all } t \in[0, T],
$$

where $\widehat{v}$ and $\widehat{\mu}$ denote the equilibrium strategy and the equilibrium aggregate distribution. In the remainder of this section, we provide a rigorous mathematical formulation of this model.

\subsection{Probabilistic Setting and Common Noise}

Throughout, we fix a time horizon $T>0$ and a finite set $\mathbb{W}$ and work on a probability space $(\Omega, \mathfrak{A}, \mathbb{P})$ that carries a finite sequence $W_{1}, \ldots, W_{n}$ of i.i.d. random variables that are uniformly distributed ${ }^{2}$ on $\mathbb{W}$. We refer to $W_{1}, \ldots, W_{n}$ as common noise factors and to $\mathbb{P}$ as the reference probability. The common noise factor $W_{k}$ is revealed at time $T_{k}$, where

$$
0 \triangleq T_{0}<T_{1}<T_{2}<\cdots<T_{n}<T_{n+1} \triangleq T
$$

Both $n$ and the common noise times $T_{0}, T_{1}, \ldots, T_{n+1}$ are fixed and deterministic. The piecewise constant filtration $\mathfrak{G}=\left\{\mathfrak{G}_{t}\right\}$ generated by common noise events is given by

$$
\mathfrak{G}_{t} \triangleq \sigma\left(W_{k}: k \in[1: n], T_{k} \leq t\right) \vee \mathfrak{N} \text { for } t \in[0, T]
$$

where $\mathfrak{N}$ denotes the set of $\mathbb{P}$-null sets. For each configuration of common noise factors $w \in \mathbb{W}^{n}$ we write

$$
w_{t} \triangleq\left(w_{1}, \ldots, w_{k}\right) \text { for } t \in\left[T_{k}, T_{k+1}\right\rangle, k \in[0: n],
$$

where for $0 \leq s \leq t \leq T$ we set $[s, t\rangle \triangleq[s, t)$ if $t<T$ and $[s, T\rangle \triangleq[s, T]$. With this convention, $W=\left\{W_{t}\right\}$ represents a piecewise constant, $\mathfrak{G}$-adapted process.

Definition 1 A function $f:[0, T] \times \mathbb{W}^{n} \rightarrow \mathbb{R}^{m}$ is non-anticipative if for all $t \in[0, T]$

$$
f(t, w)=f(t, \bar{w}) \quad \text { whenever } w, \bar{w} \in \mathbb{W}^{n} \text { are such that } w_{t}=\bar{w}_{t} .
$$

Moreover, $f$ is regular if $f(\cdot, w)$ is absolutely continuous on $\left[T_{k}, T_{k+1}\right\rangle$ for all $k \in[0: n]$.

With a slight abuse of notation, if $f:[0, T] \times \mathbb{W}^{n} \rightarrow \mathbb{R}^{m}$ is non-anticipative, we write

$$
f\left(t, w_{t}\right) \triangleq f(t, w) \text { for } w \in \mathbb{W}^{n}, t \in[0, T] .
$$

\footnotetext{
2 While the common noise factors are i.i.d. uniformly distributed under $\mathbb{P}$, the distribution of $W_{1}, \ldots, W_{n}$ in the agent's optimization problem can be modeled arbitrarily via the functions $\kappa_{1}, \ldots, \kappa_{n}$ introduced below; see also Lemma 2.
} 
Note that for $f$ regular, the one-sided limits $f\left(T_{k}-, w\right) \triangleq \lim _{t \uparrow T_{k}} f(t, w)$ exist for all $k \in[1: n], w \in \mathbb{W}^{n}$.

\subsection{Optimization Problem}

The agent's state and action spaces are given by

$$
\mathbb{S} \triangleq[1: d] \quad \text { and } \quad \mathbb{U} \subseteq \mathbb{R}^{k}, \quad \text { where } d, k \in \mathbb{N} \text { and } \mathbb{U} \neq \varnothing
$$

and we identify the space of aggregate distributions on $\mathbb{S}$ with the space of probability vectors

$$
\mathbb{M} \triangleq\left\{m \in[0, \infty)^{1 \times d}: \sum_{i=1}^{d} m^{i}=1\right\}
$$

The coefficients in the state dynamics and payoff functional are bounded and Borel measurable functions

$$
\begin{array}{lrl}
Q:[0, T] \times \mathbb{W}^{n} \times \mathbb{M} \times \mathbb{U} \rightarrow \mathbb{R}^{d \times d} & J:[0, T] \times \mathbb{W}^{n} \times \mathbb{M} \rightarrow \mathbb{S}^{d} \\
\psi:[0, T] \times \mathbb{W}^{n} \times \mathbb{M} \times \mathbb{U} \rightarrow \mathbb{R}^{d} & \Psi: \mathbb{W}^{n} \times \mathbb{M} \rightarrow \mathbb{R}^{d}
\end{array}
$$

such that $Q(\cdot, \cdot, m, u), \psi(\cdot, \cdot, m, u)$ and $J(\cdot, \cdot, m)$ are non-anticipative for all fixed $m \in$ $\mathbb{M}$ and $u \in \mathbb{U} ; Q$ satisfies the intensity matrix conditions $Q^{i j}(t, w, m, u) \geq 0, i, j \in \mathbb{S}$, $i \neq j$ and $\sum_{j \in \mathbb{S}} Q^{i j}(t, w, m, u)=0, i \in \mathbb{S}$, for $(t, w, m, u) \in[0, T] \times \mathbb{W}^{n} \times \mathbb{M} \times \mathbb{U}$ and for each $k \in[1: n]$ the function

$\kappa_{k}: \mathbb{W}^{k} \times \mathbb{M} \rightarrow[0,1], \quad\left(w_{k}, w_{1}, \ldots, w_{k-1}, m\right) \mapsto \kappa_{k}\left(w_{k} \mid w_{1}, \ldots, w_{k-1}, m\right)$,

is Borel measurable with $\sum_{\bar{w}_{k} \in \mathbb{W}} \kappa_{k}\left(\bar{w}_{k} \mid w_{1}, \ldots, w_{k-1}, m\right)=1$ for all $w_{1}, \ldots, w_{k-1} \in$ $\mathbb{W}$ and $m \in \mathbb{M}$.

We further suppose that $(\Omega, \mathfrak{A}, \mathbb{P})$ supports, for each $i, j \in \mathbb{S}, i \neq j$, a standard (i.e., unit intensity) Poisson process $N^{i j}=\left\{N_{t}^{i j}\right\}$ and an $\mathbb{S}$-valued random variable $X_{0}$ such that

$$
X_{0} \quad \text { and } \quad N^{i j}, i, j \in \mathbb{S}, i \neq j \quad \text { and } \quad W_{1}, \ldots, W_{n} \quad \text { are independent. }
$$

The corresponding full filtration $\mathfrak{F}=\left\{\mathfrak{F}_{t}\right\}$ is given by

$$
\mathfrak{F}_{t} \triangleq \sigma\left(X_{0}, W_{s}, N_{s}^{i j}: s \in[0, t] ; i, j \in \mathbb{S}, i \neq j\right) \vee \mathfrak{N} \text { for } t \in[0, T]
$$

Note that $\mathfrak{G}_{t} \subseteq \mathfrak{F}_{t}$ for all $t \in[0, T]$, that both $\mathfrak{G}$ and $\mathfrak{F}$ satisfy the usual conditions, and that $N^{i j}$ is a standard $(\mathfrak{F}, \mathbb{P})$-Poisson process for $i, j \in \mathbb{S}, i \neq j$. Given a regular, 
non-anticipative function $\mu$, the $\mathfrak{G}$-adapted, $\mathbb{M}$-valued ex ante aggregate distribution $M=\left\{M_{t}\right\}$ is given by

$$
M_{t} \triangleq \mu\left(t, W_{t}\right) \text { for } t \in[0, T]
$$

and the agent's optimization problem reads ${ }^{3}$

$$
\mathbb{E}^{v}\left[\int_{0}^{T} \psi^{X_{t}}\left(t, W_{t}, M_{t}, v_{t}\right) \mathrm{d} t+\Psi^{X_{T}}\left(W_{T}, M_{T}\right)\right] \underset{v \in \mathcal{A}}{\longrightarrow} \max !
$$

where the class of admissible strategies for $\left(\mathrm{P}_{\mu}\right)$ is given by the set of closed-loop controls

$$
\begin{aligned}
\mathcal{A} \triangleq\left\{v:[0, T] \times \mathbb{S}^{[0, T]} \times \mathbb{W}^{n} \rightarrow \mathbb{U}:\right. & v \text { is Borel measurable and } \\
& \left.v(\cdot, x, \cdot) \text { is non-anticipative for all } x \in \mathbb{S}^{[0, T]}\right\}
\end{aligned}
$$

Note that $\mathcal{A}$ subsumes the class of Markovian feedback controls considered in, e.g., [31] or [34], and that each $v \in \mathcal{A}$ canonically induces an $\mathfrak{F}$-adapted $\mathbb{U}$-valued process via

$$
v_{t} \triangleq v\left(t, X_{(\cdot \wedge t)-}, W_{t}\right) \text { for } t \in[0, T]
$$

$\mathbb{E}^{v}[\cdot]$ denotes the expectation operator with respect to the probability measure $\mathbb{P}^{v}$ given by

$$
\begin{aligned}
\frac{\mathrm{d} \mathbb{P}^{v}}{\mathrm{~d} \mathbb{P}}= & \prod_{\substack{i, j \in \mathbb{S}, i \neq j}}\left(\exp \left\{\int_{0}^{T}\left(1-Q^{i j}\left(t, W_{t}, M_{t}, v_{t}\right)\right) \mathrm{d} t\right\} \cdot \prod_{\substack{t \in(0, T], \Delta N_{t}^{i j} \neq 0}} Q^{i j}\left(t, W_{t}, M_{t}, v_{t}\right)\right) \\
& \times|\mathbb{W}|^{n} \cdot \prod_{k=1}^{n} \kappa_{k}\left(W_{k} \mid W_{1}, \ldots, W_{k-1}, M_{T_{k}-}\right)
\end{aligned}
$$

and the agent's state process $X$ is given by

$$
\mathrm{d} X_{t}=\sum_{\substack{i, j \in \mathbb{S}, i \neq j}} \mathbb{1}_{\left\{X_{t-}=i\right\}}(j-i) \mathrm{d} N_{t}^{i j} \quad \text { for } t \in\left[T_{k}, T_{k+1}\right\rangle, k \in[0: n],
$$

subject to the jump conditions

$$
X_{T_{k}}=J^{X_{T_{k}-}}\left(T_{k}, W_{T_{k}}, M_{T_{k}-}\right) \text { for } k \in[1: n] .
$$

\footnotetext{
${ }^{3}$ For notational simplicity, we write $X_{t}$ instead of $X_{t-}, M_{t}$ instead of $M_{t-}$, etc., where it does not make a difference. 
Here $N^{i j}$ triggers transitions from state $i$ to state $j$, and $\mathbb{P}^{v}$ is defined in such a way that $N^{i j}$ has $\mathbb{P}^{v}$-intensity $Q^{i j}\left(t, W_{t}, M_{t}, v_{t}\right)$; see Lemma 2 below. ${ }^{4}$ In summary, in order to formulate a mean field model within the above setting, it suffices to specify

- the agent's state space $\mathbb{S}$, action space $\mathbb{U}$ and the common noise space $\mathbb{W}$,

- the transition intensities $Q(t, w, m, u)$, transition kernels $\kappa_{k}\left(w_{k} \mid w_{1}, \ldots, w_{k-1}, m\right)$ and common noise jumps $J(t, w, m)$, and finally

- the reward functions $\psi(t, w, m, u)$ and $\Psi(w, m)$.

\subsection{State Dynamics}

In what follows, we show that the preceding construction implies the dynamics described informally above.

Lemma 2 ( $\mathbb{P}^{v}$-dynamics) For each admissible strategy $v \in \mathcal{A}, \mathbb{P}^{v}$ is a well-defined probability measure on $(\Omega, \mathfrak{A})$, absolutely continuous with respect to $\mathbb{P}$, and satisfies

$$
\mathbb{P}^{v}=\mathbb{P} \text { on } \sigma\left(X_{0}\right) \text {. }
$$

Moreover, $N^{i j}$ is a counting process with $\left(\mathfrak{F}, \mathbb{P}^{v}\right)$-intensity $\lambda^{i j}=\left\{\lambda_{t}^{i j}\right\}$, where

$$
\lambda_{t}^{i j} \triangleq Q^{i j}\left(t, W_{t}, M_{t}, v_{t}\right) \quad \text { for } t \in[0, T] \text { and } i, j \in \mathbb{S}, i \neq j
$$

Finally, for all $k \in[1: n]$ we have

$$
\mathbb{P}^{v}\left(W_{k}=w_{k} \mid \mathfrak{G}_{T_{k}-}\right)=\kappa_{k}\left(w_{k} \mid W_{1}, \ldots, W_{k-1}, M_{T_{k}-}\right) \text { for all } w_{1}, \ldots, w_{k} \in \mathbb{W}
$$

where $\mathfrak{G}$ denotes the common noise filtration and, in particular,

$$
\mathbb{P}^{v_{1}}=\mathbb{P}^{v_{2}} \text { on } \mathfrak{G}_{T} \text { for all admissible strategies } v_{1}, v_{2} \in \mathcal{A} \text {. }
$$

Proof We fix $v \in \mathcal{A}$ and split the proof into four steps.

Step 1: $\mathbb{P}^{v}$ is well-defined by (1). Since $N^{i j}$ is a standard Poisson process under $\mathbb{P}$, the compensated process $\bar{N}_{t}^{i j} \triangleq N_{t}^{i j}-t, t \geq 0$, is an $(\mathfrak{F}, \mathbb{P})$-martingale for all $i, j \in \mathbb{S}, i \neq j$. We define $\theta^{v}=\left\{\theta_{t}^{v}\right\} \operatorname{via}^{5}$

$$
\theta_{t}^{v} \triangleq \sum_{\substack{i, j \in \mathbb{S}, i \neq j}} \int_{0}^{t}\left(Q^{i j}\left(s, W_{s}, \mu\left(s, W_{s}\right), v_{s}\right)-1\right) \mathrm{d} \bar{N}_{s}^{i j}, \quad t \in[0, T]
$$

\footnotetext{
${ }^{4}$ See also [7, Sect. 3.3] for a similar change-of-measure construction of jump processes with stochastic intensities.

${ }^{5}$ Note that $\left.\left.\int_{0}^{t} Q^{i j}\left(s, W_{s}, \mu\left(s, W_{s}\right), v_{s}\right)-1\right) \mathrm{d} \bar{N}_{s}^{i j}=\int_{0}^{t} Q^{i j}\left(s, W_{s-}, \mu\left(s, W_{s-}\right), v_{s}\right)-1\right) \mathrm{d} \bar{N}_{s}^{i j} \mathbb{P}_{\text {-a.s. }}$
} 
and observe that the Doléans-Dade exponential $\mathcal{E}\left[\theta^{\nu}\right]$ is a local $(\mathfrak{F}, \mathbb{P})$-martingale with

$$
\mathcal{E}\left[\theta^{v}\right]_{t}=\prod_{\substack{i, j \in \mathbb{S}, i \neq j}}\left(\exp \left\{\int_{0}^{t}\left(1-Q^{i j}\left(s, W_{s}, \mu\left(s, W_{s}\right), v_{s}\right)\right) \mathrm{d} s\right\} \cdot \prod_{\substack{s \in(0, t], \Delta N_{s}^{i j} \neq 0}} Q^{i j}\left(s, W_{s}, \mu\left(s, W_{s}\right), v_{s}\right)\right)
$$

for $t \in[0, T]$. Next, we define $\vartheta=\left\{\vartheta_{t}\right\}$ via

$$
\vartheta_{t} \triangleq \sum_{\substack{k \in[1: n], T_{k} \leq t}}\left(|\mathbb{W}| \cdot \kappa_{k}\left(W_{k} \mid W_{1}, \ldots, W_{k-1}, \mu\left(T_{k}-, W_{T_{k}-}\right)\right)-1\right), \quad t \in[0, T]
$$

and note that $\vartheta$ is an $(\mathfrak{F}, \mathbb{P})$-martingale. Indeed, for each $k \in[0: n]$ we have $\vartheta_{t}=\vartheta_{T_{k}}$ for $t \in\left[T_{k}, T_{k+1}\right\rangle$ and, using that $W_{k}$ is independent of $\mathfrak{F}_{T_{k}-}$ and uniformly distributed on $\mathbb{W}$ under $\mathbb{P}$, it follows that

$$
\begin{aligned}
& \mathbb{E}\left[\vartheta_{T_{k}} \mid \mathfrak{F}_{T_{k}-}\right]= \vartheta_{T_{k}-}+\mathbb{E}\left[|\mathbb{W}| \cdot \kappa_{k}\left(W_{k} \mid W_{1}, \ldots, W_{k-1}, \mu\left(T_{k}-, W_{T_{k}-}\right)\right)-1 \mid \mathfrak{F}_{T_{k}-}\right] \\
&=\vartheta_{T_{k}-}-1+|\mathbb{W}| \cdot \sum_{w_{k} \in \mathbb{W}} \mathbb{P}\left(W_{k}=w_{k} \mid W_{1}, \ldots, W_{k-1}, \mu\left(T_{k}-, W_{T_{k}-}\right)\right) \\
& \quad \times \kappa_{k}\left(w_{k} \mid W_{1}, \ldots, W_{k-1}, \mu\left(T_{k}-, W_{T_{k}-}\right)\right) \\
&=\vartheta_{T_{k}-}-1+|\mathbb{W}| \cdot \sum_{w_{k} \in \mathbb{W}} \frac{1}{|\mathbb{W}|} \cdot \kappa_{k}\left(w_{k} \mid W_{1}, \ldots, W_{k-1}, \mu\left(T_{k}-, W_{T_{k}-}\right)\right)=\vartheta_{T_{k}-} .
\end{aligned}
$$

Hence the Doléans-Dade exponential $\mathcal{E}[\vartheta]$ is a local $(\mathfrak{F}, \mathbb{P})$-martingale, and we have

$$
\mathcal{E}[\vartheta]_{t}=\prod_{s \in(0, t]}\left(1+\Delta \vartheta_{s}\right)=\prod_{\substack{k \in[1: n] \\ T_{k} \leq t}}\left(|\mathbb{W}| \cdot \kappa_{k}\left(W_{k} \mid W_{1}, \ldots, W_{k-1}, \mu\left(T_{k}-, W_{T_{k}-}\right)\right)\right)
$$

for $t \in[0, T]$. Since $\Delta N_{T_{k}}^{i j}=0$ for all $i, j \in \mathbb{S}, i \neq j$, and $k \in[1: n]$ a.s., we have $\left[\theta^{v}, \vartheta\right]=0$, and thus the process $Z^{v} \triangleq \mathcal{E}\left[\theta^{v}+\vartheta\right]=\mathcal{E}\left[\theta^{v}\right] \cdot \mathcal{E}[\vartheta]$, i.e.

$$
\begin{aligned}
Z_{t}^{v}= & \prod_{\substack{i, j \in \mathbb{S}, i \neq j}}\left(\exp \left\{\int_{0}^{t}\left(1-Q^{i j}\left(s, W_{s}, \mu\left(s, W_{s}\right), v_{s}\right)\right) \mathrm{d} s\right\} \cdot \prod_{\substack{s \in(0, t], \Delta N_{s}^{i j} \neq 0}} Q^{i j}\left(s, W_{s}, \mu\left(s, W_{s}\right), v_{s}\right)\right) \\
& \times \prod_{\substack{k \in[1: n], T_{k} \leq t}}\left(|\mathbb{W}| \cdot \kappa_{k}\left(W_{k} \mid W_{1}, \ldots, W_{k-1}, \mu\left(T_{k}-, W_{T_{k}-}\right)\right)\right)
\end{aligned}
$$

is a local $(\mathfrak{F}, \mathbb{P})$-martingale. Since

$$
\sup _{t \in[0, T]}\left|\mathcal{E}\left[\theta^{\nu}\right]_{t}\right| \leq \mathrm{e}^{d^{2} T} \cdot \ell^{Y}
$$


where $\ell \triangleq \max _{i, j \in \mathbb{S}, i \neq j}\left\|Q^{i j}\right\|_{\infty}$ and $Y \triangleq \sum_{i, j \in \mathbb{S}, i \neq j} N_{T}^{i j} \sim_{\mathbb{P}} \operatorname{Poisson}(d(d-1) T)$ and

$$
\sup _{t \in[0, T]}\left|\mathcal{E}[\vartheta]_{t}\right| \leq|\mathbb{W}|^{n}
$$

it follows that $\sup _{t \in[0, T]}\left|Z_{t}^{v}\right|$ is $\mathbb{P}$-integrable, so $Z^{v}$ is in fact an $(\mathfrak{F}, \mathbb{P})$-martingale. Since $Z^{v}$ is non-negative with $Z_{0}^{v}=1$ by construction, we conclude that $\mathbb{P}^{v}$ is a well-defined probability measure on $\mathfrak{A}$, absolutely continuous with respect to $\mathbb{P}$, with density process

$$
\left.\frac{\mathrm{d} \mathbb{P}^{v}}{\mathrm{~d} \mathbb{P}}\right|_{\mathfrak{F}_{t}}=Z_{t}^{v}, \quad t \in[0, T]
$$

Step 2: $\mathbb{P}^{v}$-intensity of $N^{i j}$. Let $i, j \in \mathbb{S}$ with $i \neq j$. Since $\mathbb{P}^{v} \ll \mathbb{P}$ it is clear that $N^{i j}$ is a $\mathbb{P}^{v}$-counting process, so it suffices to show that the process ${ }^{\nu} \bar{N}^{i j}=\left\{{ }^{\nu} \bar{N}_{t}^{i j}\right\}$ given by

$$
{ }^{v} \bar{N}_{t}^{i j} \triangleq N_{t}^{i j}-\int_{0}^{t} Q^{i j}\left(s, W_{s}, \mu\left(s, W_{s}\right), v_{s}\right) \mathrm{d} s, \quad t \in[0, T],
$$

is a local $\left(\mathfrak{F}, \mathbb{P}^{v}\right)$-martingale. To show this, by Step 1 it suffices to demonstrate that $Z^{v} \cdot{ }^{v} \bar{N}^{i j}$ is a local $(\mathfrak{F}, \mathbb{P})$-martingale. Noting that

- $\left[N^{k \ell}, N^{i j}\right]=\sum_{s \in(0, \cdot]} \Delta N_{s}^{k \ell} \cdot \Delta N_{s}^{i j}=0$ whenever $k, \ell \in \mathbb{S}$ and $(k, \ell) \neq(i, j)$,

- $\mathrm{d} Z_{t}^{v}=Z_{t-}^{v} \mathrm{~d} \theta_{t}^{v}+Z_{t-}^{v} \mathrm{~d} \vartheta_{t}=\sum_{k, \ell \in \mathbb{S}, k \neq \ell} Z_{t-}^{v}\left(Q^{k \ell}\left(t, W_{t}, \mu\left(t, W_{t}\right), v_{t}\right)-1\right) \mathrm{d} \bar{N}_{t}^{k \ell}$ $+Z_{t-}^{v} \mathrm{~d} \vartheta_{t}$,

- $\mathrm{d}\left[Z^{v},{ }^{v} \bar{N}^{i j}\right]_{t}=Z_{t-}^{v}\left(Q^{i j}\left(t, W_{t}, \mu\left(t, W_{t}\right), v_{t}\right)-1\right) \mathrm{d} N_{t}^{i j}$,

and using integration by parts, the local martingale property follows since

$$
\begin{aligned}
\mathrm{d}\left(Z_{t}^{v} \cdot{ }^{v} \bar{N}_{t}^{i j}\right)= & Z_{t-}^{v} \mathrm{~d}{ }^{v} \bar{N}_{t}^{i j}+{ }^{v} \bar{N}_{t-}^{i j} \mathrm{~d} Z_{t}^{v}+\mathrm{d}\left[Z^{v},{ }^{v} \bar{N}^{i j}\right] t \\
= & Z_{t-}^{v} \mathrm{~d} N_{t}^{i j}-Z_{t-}^{v} Q^{i j}\left(t, W_{t}, \mu\left(t, W_{t}\right), v_{t}\right) \mathrm{d} t+{ }^{v} \bar{N}_{t-}^{i j} \mathrm{~d} Z_{t}^{v} \\
& +Z_{t-}^{v} Q^{i j}\left(t, W_{t}, \mu\left(t, W_{t}\right), v_{t}\right) \mathrm{d} N_{t}^{i j}-Z_{t-}^{v} \mathrm{~d} N_{t}^{i j} \\
= & { }^{v} \bar{N}_{t-}^{i j} \mathrm{~d} Z_{t}^{v}+Z_{t-}^{v} Q^{i j}\left(t, W_{t}, \mu\left(t, W_{t}\right), v_{t}\right) \mathrm{d} \bar{N}_{t}^{i j} .
\end{aligned}
$$

Step 3: $\mathbb{P}^{v}=\mathbb{P}$ on $\sigma\left(X_{0}\right)$. For any function $g: \mathbb{S} \rightarrow \mathbb{R}$ we have

$\mathbb{E}^{v}\left[g\left(X_{0}\right)\right]=\mathbb{E}\left[g\left(X_{0}\right) \cdot Z_{T}^{v}\right]=\mathbb{E}\left[g\left(X_{0}\right) \cdot \mathbb{E}\left[Z_{T}^{v} \mid \mathfrak{F}_{0}\right]\right]=\mathbb{E}\left[g\left(X_{0}\right) \cdot Z_{0}^{v}\right]=\mathbb{E}\left[g\left(X_{0}\right)\right]$

by the $(\mathfrak{F}, \mathbb{P})$-martingale property of $Z^{v}$. 
Step 4: Distribution of $W_{k}$ under $\mathbb{P}^{v}$. Let $k \in[1: n]$ and $w_{1}, \ldots, w_{k} \in \mathbb{W}$. Since $\mathcal{E}\left[\theta^{v}\right]_{T_{k}}=\mathcal{E}\left[\theta^{\nu}\right]_{T_{k}}$ a.s. and $W_{k}$ is uniformly distributed on $\mathbb{W}$ and independent of $\mathfrak{F}_{T_{k}-}$ under $\mathbb{P}$, iterated conditioning yields

$$
\begin{gathered}
\mathbb{P}^{v}\left(W_{1}=w_{1}, \ldots, W_{k}=w_{k}\right)=\mathbb{E}\left[Z_{T_{k}}^{v} \cdot \mathbb{1}_{\left\{W_{k}=w_{k}\right\}} \cdot \mathbb{1}_{\left\{W_{1}=w_{1}, \ldots, W_{k-1}=w_{k-1}\right\}}\right] \\
=\mathbb{E}\left[Z_{T_{k}-}^{v} \cdot|\mathbb{W}| \cdot \kappa_{k}\left(W_{k} \mid W_{1}, \ldots, W_{k-1}, \mu\left(T_{k}-, W_{T_{k}-}\right)\right) \cdot \mathbb{1}_{\left\{W_{k}=w_{k}\right\}} \cdot \mathbb{1}_{\left\{W_{1}=w_{1}, \ldots, W_{k-1}=w_{k-1}\right\}}\right] \\
=|\mathbb{W}| \cdot \kappa_{k}\left(w_{k} \mid w_{1}, \ldots, w_{k-1}, \mu\left(T_{k}-, w_{T_{k}-}\right)\right) \cdot \mathbb{E}\left[Z_{T_{k}-}^{v} \cdot \mathbb{1}_{\left\{W_{1}=w_{1}, \ldots, W_{k-1}=w_{k-1}\right\}}\right. \\
\left.\cdot \mathbb{P}\left(W_{k}=w_{k} \mid \mathfrak{F}_{T_{k}-}\right)\right] \\
=\kappa_{k}\left(w_{k} \mid w_{1}, \ldots, w_{k-1}, \mu\left(T_{k}-, w_{T_{k}-}\right)\right) \cdot \mathbb{P}^{v}\left(W_{1}=w_{1}, \ldots, W_{k-1}=w_{k-1}\right) .
\end{gathered}
$$

Thus we have $\mathbb{P}^{v}\left(W_{k}=w_{k} \mid \mathfrak{G}_{T_{k}-}\right)=\kappa_{k}\left(w_{k} \mid W_{1}, \ldots, W_{k-1}, M_{T_{k}-}\right)$ and the proof is complete.

Lemma 2 implies in particular that $\mathbb{P}^{v}\left(\Delta N_{t}^{i j} \neq 0\right)=0$ for every $t \in[0, T]$, so as a consequence we have

$$
\Delta X_{t}=0 \quad \mathbb{P}^{v} \text {-a.s. for all } t \in[0, T] \backslash\left\{T_{1}, \ldots, T_{n}\right\}
$$

Moreover, since $\mathbb{P}^{v_{1}}=\mathbb{P}^{v_{2}}$ on $\mathfrak{G}_{T}$ for all admissible controls $v_{1}, v_{2} \in \mathcal{A}$ and $M_{t}=$ $\mu\left(t, W_{t}\right)$ for $t \in[0, T]$, the agent's ex ante beliefs concerning the common noise factors are the same, irrespective of his control.

\section{Solution of the Optimization Problem}

In the following, we solve the agent's maximization problem $\left(\mathrm{P}_{\mu}\right)$ using the associated dynamic programming equation (DPE). This is the same methodology as in [31] and [15]; see [22] for an alternative approach (to extended mean field games, but without common noise) based on backward SDEs.

The DPE for the value function of the agent's optimization problem $\left(\mathrm{P}_{\mu}\right)$ reads

$$
0=\sup _{u \in \mathbb{U}}\left\{\frac{\partial v^{i}}{\partial t}(t, w)+\psi^{i}(t, w, \mu(t, w), u)+Q^{i \cdot}(t, w, \mu(t, w), u) \cdot v(t, w)\right\}
$$

for $i \in \mathbb{S}$, subject to suitable consistency conditions for $t=T_{k}, k \in[1: n]$, and the terminal condition

$$
v(T, w)=\Psi(w, \mu(T, w)) \text { for all } w \in \mathbb{W}^{n} .
$$

Assumption 3 There exists a Borel measurable function $h:[0, T] \times \mathbb{W}^{n} \times \mathbb{M} \times \mathbb{R}^{d} \rightarrow$ $\mathbb{U}^{d}$ such that for every $i \in \mathbb{S}$ and all $(t, w, m, v) \in[0, T] \times \mathbb{W}^{n} \times \mathbb{M} \times \mathbb{R}^{d}$ we have

$$
h^{i}(t, w, m, v) \in \underset{u \in \mathbb{U}}{\arg \max }\left\{\psi^{i}(t, w, m, u)+Q^{i \cdot}(t, w, m, u) \cdot v\right\} .
$$


Assumption 3 is satisfied e.g. if $\mathbb{U}$ is compact and $Q$ and $\psi$ are continuous with respect to $u \in \mathbb{U}$. Note that, since $\psi^{i}(\cdot, \cdot, m, u)$ and $Q^{i \cdot}(\cdot, \cdot, m, u)$ are non-anticipative for $m \in \mathbb{M}, u \in \mathbb{U}$, we can assume without loss of generality that $h(\cdot, \cdot, m, v)$ is non-anticipative for $m \in \mathbb{M}, v \in \mathbb{R}^{d}$. With this, we define

$$
\begin{array}{ll}
\widehat{Q}:[0, T] \times \mathbb{W}^{n} \times \mathbb{M} \times \mathbb{R}^{d} \rightarrow \mathbb{R}^{d \times d}, & \widehat{Q}^{i j}(t, w, m, v) \triangleq Q^{i j}\left(t, w, m, h^{i}(t, w, m, v)\right), \\
\widehat{\psi}:[0, T] \times \mathbb{W}^{n} \times \mathbb{M} \times \mathbb{R}^{d} \rightarrow \mathbb{R}^{d}, & \widehat{\psi}^{i}(t, w, m, v) \triangleq \psi^{i}\left(t, w, m, h^{i}(t, w, m, v)\right)
\end{array}
$$

and thus obtain the following reduced-form DPE, which we use in the following:

Definition 4 Let $\mu:[0, T] \times \mathbb{W}^{n} \rightarrow \mathbb{M}$ be regular and non-anticipative. A function $v:[0, T] \times \mathbb{W}^{n} \rightarrow \mathbb{R}^{d}$ is called a solution of $\left(\mathrm{DP}_{\mu}\right)$ subject to $\left(\mathrm{CC}_{\mu}\right),\left(\mathrm{TC}_{\mu}\right)$ if $v$ is non-anticipative and satisfies the ordinary differential equation $(\mathrm{ODE})^{6}$

$$
\dot{v}(t, w)=-\widehat{\psi}(t, w, \mu(t, w), v(t, w))-\widehat{Q}(t, w, \mu(t, w), v(t, w)) \cdot v(t, w) \quad\left(\mathrm{DP}_{\mu}\right)
$$

for $t \in\left[T_{k}, T_{k+1}\right\rangle, k \in[0: n]$, subject to the consistency and terminal conditions

$$
\begin{gathered}
v\left(T_{k}-, w\right)=\Psi_{k}\left(w, \mu\left(T_{k}-, w\right), v\left(T_{k}, \cdot\right)\right), \\
v(T, w)=\Psi(w, \mu(T, w))
\end{gathered}
$$

for $k \in[1: n]$ and all $w \in \mathbb{W}^{n}$. Here, for $k \in[1: n]$, the jump operator $\Psi_{k}$ is defined via

$$
\Psi_{k}^{i}(w, m, \bar{v}) \triangleq \sum_{\bar{w}_{k} \in \mathbb{W}} \kappa_{k}\left(\bar{w}_{k} \mid w_{1}, \ldots, w_{k-1}, m\right) \cdot \bar{v}^{J^{i}\left(T_{k},\left(w_{-k}, \bar{w}_{k}\right), m\right)}\left(w_{-k}, \bar{w}_{k}\right), i \in \mathbb{S},
$$

where $\bar{v}: \mathbb{W}^{n} \rightarrow \mathbb{R}^{d}$ and $\left(w_{-k}, \bar{w}_{k}\right) \triangleq\left(w_{1}, \ldots, w_{k-1}, \bar{w}_{k}, w_{k+1}, \ldots, w_{n}\right)$ for $\bar{w}_{k} \in \mathbb{W}, w \in \mathbb{W}^{n}$.

Observe that $\left(\mathrm{DP}_{\mu}\right)$ represents a system of (random) ODEs, coupled via $w \in \mathbb{W}^{n}$. The ODEs run backward in time on each segment $\left[T_{k}, T_{k+1}\right\rangle \times \mathbb{W}^{n}, k \in[0: n]$, and their terminal conditions for $t \uparrow T_{k+1}$ are specified by $\left(\mathrm{TC}_{\mu}\right)$ for $k=n$ and by $\left(\mathrm{CC}_{\mu}\right)$ for $k<n$. Note that for $t \in\left[T_{k}, T_{k+1}\right\rangle$ the relevant common noise factors $W_{1}, \ldots, W_{k}$ are known.

Remark 5 While the significance of the $\mathrm{DPE}\left(\mathrm{DP}_{\mu}\right)$ and the terminal condition $\left(\mathrm{TC}_{\mu}\right)$ are clear, the consistency conditions $\left(\mathrm{CC}_{\mu}\right)$ warrant a brief comment: For $i \in \mathbb{S}$, $k \in[1: n]$ and $w \in \mathbb{W}^{n}$ the state process jumps from state $i$ to state $j \triangleq$ $J^{i}\left(T_{k},\left(w_{-k}, W_{k}\right), \mu\left(T_{k}-, w_{T_{k}-}\right)\right)$ on $\left\{X_{T_{k}-}=i\right\} \cap\left\{W_{T_{k}-}=w_{T_{k}-}\right\}$ when the common noise factor $W_{k}$ is revealed at time $T_{k}$.

\footnotetext{
${ }^{6}$ All ODEs in this article are taken in the sense of Carathéodory; see [36, §I.5]. Briefly, $x: I \rightarrow \mathbb{R}$ is a solution of $\dot{x}(t)=f(t, x(t)), x\left(t_{0}\right)=x_{0}$, in the sense of Carathéodory if $t \mapsto f(t, x(t))$ is Lebesgue integrable and satisfies $x(t)=x_{0}+\int_{t_{0}}^{t} f(s, x(s)) \mathrm{d} s$ for all $t \in I$.
} 
We next link the solution of the DPE to the underlying stochastic control problem.

Theorem 6 (Verification) Suppose $\mu:[0, T] \times \mathbb{W}^{n} \rightarrow \mathbb{M}$ is regular and nonanticipative and $v$ is a solution of $\left(D P_{\mu}\right)$ subject to $\left(C C_{\mu}\right)$ and $\left(T C_{\mu}\right)$. Then $v$ is the agent's value function for problem $\left(P_{\mu}\right)$, i.e.

$$
\sum_{i \in \mathbb{S}} \mathbb{P}\left(X_{0}=i\right) v^{i}(0)=\sup _{v \in \mathcal{A}} \mathbb{E}^{\nu}\left[\int_{0}^{T} \psi^{X_{t}}\left(t, W_{t}, M_{t}, v_{t}\right) \mathrm{d} t+\Psi^{X_{T}}\left(W_{T}, M_{T}\right)\right]
$$

and an optimal control is given by $\widehat{v} \in \mathcal{A}$ with

$$
\widehat{v}\left(t, X_{(\cdot \wedge t)_{-},}, W_{t}\right)=h^{X_{t-}}\left(t, W_{t}, \mu\left(t, W_{t}\right), v\left(t, W_{t}\right)\right) \text { for } t \in[0, T]
$$

Proof Let $v \in \mathcal{A}$ be an admissible strategy. Until further notice we fix $k \in[0: n]$.

Step 1: Dynamics on $\left[T_{k}, T_{k+1}\right\rangle$. From Itô's lemma, applicable due to regularity of $v$, we obtain

$$
\begin{aligned}
v^{X_{T_{k}}}\left(T_{k}, W_{T_{k}}\right)= & v^{X_{T_{k+1}-}}\left(T_{k+1}-, W_{T_{k+1}-}\right) \\
& -\sum_{\substack{i, j \in \mathbb{S}, i \neq j}} \int_{T_{k}}^{T_{k+1}} \mathbb{1}_{\left\{X_{s-}=i\right\}}\left(v^{j}\left(s, W_{s}\right)-v^{i}\left(s, W_{s}\right)\right) \mathrm{d}^{v} \bar{N}_{s}^{i j} \\
& -\int_{T_{k}}^{T_{k+1}} \sum_{i=1}^{d} \mathbb{1}_{\left\{X_{s}=i\right\}}\left(\dot{v}^{i}\left(s, W_{s}\right)+Q^{i \cdot}\left(s, W_{s}, \mu\left(s, W_{s}\right), v_{s}\right) \cdot v\left(s, W_{s}\right)\right) \mathrm{d} s .
\end{aligned}
$$

Step 2: Jump dynamics at $T_{k}$. We recall from Lemma 2 that

$$
\begin{aligned}
\mathbb{P}^{v}\left(W_{k}=\bar{w}_{k} \mid X_{T_{k}-}, W_{1}, \ldots, W_{k-1}\right) & =\mathbb{P}^{v}\left(W_{k}=\bar{w}_{k} \mid W_{1}, \ldots, W_{k-1}\right) \\
& =\kappa_{k}\left(\bar{w}_{k} \mid W_{1}, \ldots, W_{k-1}, \mu\left(T_{k}-, W_{T_{k}-}\right)\right) .
\end{aligned}
$$

In view of the jump dynamics (3) and the consistency condition $\left(\mathrm{CC}_{\mu}\right)$, we thus obtain

$$
\begin{aligned}
\mathbb{E}^{v} & {\left[v^{X_{T_{k}}}\left(T_{k}, W_{T_{k}}\right) \mid \sigma\left(X_{T_{k}-}, W_{T_{k}-}\right)\right] } \\
& =\mathbb{E}^{v}\left[v^{J^{X} T_{T_{k}-}\left(T_{k},\left(W_{T_{k}-}, W_{k}\right), \mu\left(T_{k}-, W_{T_{k}-}\right)\right)}\left(T_{k},\left(W_{T_{k}-}, W_{k}\right)\right) \mid X_{T_{k}-}, W_{T_{k}-}\right] \\
& =\sum_{\bar{w}_{k} \in \mathbb{W}} \kappa_{k}\left(\bar{w}_{k} \mid W_{T_{k}-}, \mu\left(T_{k}-, W_{T_{k}-}\right)\right) v^{J^{X_{T_{k}-}}\left(T_{k},\left(W_{T_{k}-}, \bar{w}_{k}\right), \mu\left(T_{k}-, W_{T_{k}-}\right)\right)}\left(T_{k},\left(W_{T_{k}-}, \bar{w}_{k}\right)\right) \\
& =\Psi_{k}^{X_{T_{k}-}}\left(W_{T_{k}-}, \mu\left(T_{k}-, W_{T_{k}-}\right), v\left(T_{k}, \cdot\right)\right)=v^{X_{T_{k}-}}\left(T_{k}-, W_{T_{k}-}\right) .
\end{aligned}
$$

Step 3: Optimality. Combining (11) and (12) for $k=[1: n]$ and using $\left(\mathrm{TC}_{\mu}\right)$ yields 


$$
\begin{aligned}
v^{X_{0}}(0)= & v^{X_{T}}\left(T, W_{T}\right)+\sum_{k=1}^{n}\left(v^{X_{T_{k}}}\left(T_{k}-, W_{T_{k}-}\right)-v^{X_{T_{k}}}\left(T_{k}, W_{T_{k}}\right)\right) \\
& -\sum_{\substack { k=0 \\
\begin{subarray}{c}{i, j \in \mathbb{S}, i \neq j{ k = 0 \\
\begin{subarray} { c } { i , j \in \mathbb { S } , \\
i \neq j } }\end{subarray}} \int_{T_{k}}^{T_{k+1}} \mathbb{1}_{\left\{X_{s-}=i\right\}}\left(v^{j}\left(s, W_{s}\right)-v^{i}\left(s, W_{s}\right)\right) \mathrm{d}^{v} \bar{N}_{s}^{i j} \\
& \left.+\int_{T_{k}}^{T_{k+1}} \sum_{i=1}^{d} \mathbb{1}_{\left\{X_{s}=i\right\}}\left(\dot{v}^{i}\left(s, W_{s}\right)+Q^{i \cdot}\left(s, W_{s}, \mu\left(s, W_{s}\right), v_{s}\right) \cdot v\left(s, W_{s}\right)\right) \mathrm{d} s\right] \\
= & \Psi^{X_{T}}\left(W_{T}, \mu\left(T, W_{T}\right)\right)+\sum_{k=1}^{n}\left(\mathbb{E}^{v}\left[v^{X_{T_{k}}}\left(T_{k}, W_{T_{k}}\right) \mid \sigma\left(X_{T_{k}-}, W_{T_{k}-}\right)\right]-v^{X_{T_{k}}}\left(T_{k}, W_{T_{k}}\right)\right) \\
& -\sum_{\substack{i, j \in \mathbb{S}, i \neq j}} M_{T}^{i j}-\int_{0}^{T} \sum_{i=1}^{d} \mathbb{1}_{\left\{X_{s}=i\right\}}\left(\dot{v}^{i}\left(s, W_{s}\right)+Q^{i \cdot}\left(s, W_{s}, \mu\left(s, W_{s}\right), v_{s}\right) \cdot v\left(s, W_{s}\right)\right) \mathrm{d} s,
\end{aligned}
$$

where for $i, j \in \mathbb{S}, i \neq j$ the local $\left(\mathfrak{F}, \mathbb{P}^{v}\right)$-martingale $M^{i j}$ is given by

$$
M_{t}^{i j} \triangleq \int_{0}^{t} \mathbb{1}_{\left\{X_{s-}=i\right\}}\left(v^{j}\left(s, W_{s}\right)-v^{i}\left(s, W_{s}\right)\right) \mathrm{d}^{v} \bar{N}_{s}^{i j} \quad \text { for } t \in[0, T]
$$

Since ${ }^{v} \bar{N}^{i j}$ is a compensated counting process and $v$ and $Q$ are bounded, $M^{i j}$ is in fact an $\left(\mathfrak{F}, \mathbb{P}^{v}\right)$-martingale. Hence taking $\mathbb{P}^{v}$-expectations in (13), using the tower property of conditional expectation and the fact that $\mathbb{P}^{v}$ and $\mathbb{P}$ coincide on $\sigma\left(X_{0}\right)$ by Lemma 2 , and finally that $v$ solves the DPE, we obtain

$$
\begin{aligned}
\sum_{i \in \mathbb{S}} \mathbb{P}\left(X_{0}=i\right) v^{i}(0)= & \mathbb{E}\left[v^{X_{0}}(0)\right]=\mathbb{E}^{v}\left[v^{X_{0}}(0)\right] \\
= & \mathbb{E}^{v}\left[\Psi^{X_{T}}\left(W_{T}, \mu\left(T, W_{T}\right)\right)\right. \\
& \left.-\int_{0}^{T} \sum_{i=1}^{d} \mathbb{1}_{\left\{X_{s}=i\right\}}\left(\dot{v}^{i}\left(s, W_{s}\right)+Q^{i \cdot}\left(s, W_{s}, \mu\left(s, W_{s}\right), v_{s}\right) \cdot v\left(s, W_{s}\right)\right) \mathrm{d} s\right] \\
\geq & \mathbb{E}^{v}\left[\Psi^{X_{T}}\left(W_{T}, M_{T}\right)+\int_{0}^{T} \psi^{X_{s}}\left(s, W_{s}, M_{s}, v_{s}\right) \mathrm{d} s\right]
\end{aligned}
$$

If we replace $v$ with $\widehat{v}$, the same argument applies with equality in (14); we thus conclude that $v$ is the value function of $\left(\mathrm{P}_{\mu}\right)$, and that the strategy $\widehat{v}$ is optimal.

The optimal strategy is Markovian in the agent's state; this is unsurprising given the literature, see e.g. [31, Theorem 1] or [22, Proposition 3.9] and [15, Theorem 4]. Note, however, that the time- $t$ optimal strategy may depend on all common noise events that have occurred up to time $t$, as $W_{t}=\left(W_{1}, \ldots, W_{k}\right)$ for $t \in\left[T_{k}, T_{k+1}\right\rangle$. In the following, we denote by $\widehat{\mathbb{P}}$ the probability measure

$$
\widehat{\mathbb{P}} \triangleq \mathbb{P}^{\hat{v}}
$$


where $\widehat{v}$ is the optimal control specified in Theorem 6. It follows from Lemma 2 that $N^{i j}$ has $\widehat{\mathbb{P}}$-intensity $\widehat{\lambda}^{i j}=\left\{\widehat{\lambda}_{t}^{i j}\right\}$ for $i, j \in \mathbb{S}, i \neq j$, where

$$
\widehat{\lambda}_{t}^{i j} \triangleq Q^{i j}\left(t, W_{t}, \mu\left(t, W_{t}\right), h^{X_{t-}}\left(t, W_{t}, \mu\left(t, W_{t}\right), v\left(t, W_{t}\right)\right)\right) \text { for } t \in[0, T]
$$

\section{Equilibrium}

Having solved the agent's optimization problem for a given ex ante function $\mu$, we now turn to the resulting mean field equilibrium. We first identify the aggregate distribution resulting from the optimal control.

Remark 7 This paper generally adopts a "representative agent" point of view; an alternative justification of mean field equilibrium is via convergence of Nash equilibria of symmetric $N$-player games in the limit $N \rightarrow \infty$; see, among others, $[2,14,15,18,20,22,24,28]$. In the setting of this article (albeit under additional regularity conditions) a mean field limit justification can be provided along the lines of the proof of Theorem 7 in [31] by conditioning on common noise configurations, similarly as in the proof of Theorem 9 below.

\subsection{Aggregation}

Given an ex ante aggregate distribution specified in terms of a regular, non-anticipative function $\mu$ and a corresponding solution $v$ of $\left(\mathrm{DP}_{\mu}\right)$ subject to $\left(\mathrm{CC}_{\mu}\right),\left(\mathrm{TC}_{\mu}\right)$, Theorem 6 yields an optimal strategy $\widehat{v}$ for the agent's optimization problem $\left(\mathrm{P}_{\mu}\right)$. With $\widehat{\mathbb{P}}$ denoting the probability measure associated with $\widehat{v}$, the resulting ex post aggregate distribution is given by the $\mathbb{M}$-valued, $\mathfrak{G}$-adapted process $\widehat{M}=\left\{\widehat{M}_{t}\right\}$,

$$
\widehat{M}_{t} \triangleq \widehat{\mathbb{P}}\left(X_{t} \in \cdot \mid \mathfrak{G}_{t}\right) \quad \text { for } t \in[0, T]
$$

where $\mathfrak{G}$ denotes the common noise filtration. We note that $\widehat{M}$ is càdlàg since $\mathfrak{G}$ is piecewise constant and $X$ is càdlàg. Equilibrium obtains if $\widehat{M}_{t}=\mu\left(t, W_{t}\right)$ for all $t \in[0, T]$. To proceed, we aim for a more explicit description of $\widehat{M}$ and, in particular, its dynamics. Thus we define for $k \in[1: n]$

$$
\Phi_{k}: \mathbb{W}^{n} \times \mathbb{M} \times \mathbb{M} \rightarrow \mathbb{M}, \quad \Phi_{k}(w, m, \bar{m}) \triangleq m \cdot P_{k}(w, \bar{m}),
$$

where $P_{k}: \mathbb{W}^{n} \times \mathbb{M} \rightarrow\{0,1\}^{d \times d}$ is given by

$$
P_{k}^{i j}(w, \bar{m}) \triangleq \mathbb{1}_{\left\{J^{i}\left(T_{k}, w_{1}, \ldots, w_{k}, \bar{m}\right)=j\right\}} \quad \text { for } i, j \in \mathbb{S}
$$

and we set

$$
m_{0} \triangleq \mathbb{P}\left(X_{0} \in \cdot\right)=\widehat{\mathbb{P}}\left(X_{0} \in \cdot\right) \in \mathbb{M}
$$


Lemma 8 Let $\mu:[0, T] \times \mathbb{W}^{n} \rightarrow \mathbb{M}$ and $v:[0, T] \times \mathbb{W}^{n} \rightarrow \mathbb{R}^{d}$ be regular and non-anticipative, and suppose that $Y=\left\{Y_{t}\right\}$ is an $\mathbb{M}$-valued stochastic process with dynamics

$$
\begin{aligned}
& Y_{0}=m_{0}, \quad Y_{t}=Y_{T_{k}}+\int_{T_{k}}^{t} Y_{s} \cdot \widehat{Q}\left(s, W_{s}, \mu\left(s, W_{s}\right), v\left(s, W_{s}\right)\right) \mathrm{d} s \\
& \text { for } t \in\left[T_{k}, T_{k+1}\right\rangle, k \in[0: n]
\end{aligned}
$$

that satisfies the consistency conditions

$$
Y_{T_{k}}=\Phi_{k}\left(W_{T_{k}}, Y_{T_{k}-}, \mu\left(T_{k}-, W_{T_{k}-}\right)\right) \text { for } k \in[1: n]
$$

Then $Y$ is $\mathfrak{G}$-adapted.

Proof Step 1: Existence and uniqueness of Carathéodory solutions. For each $k \in$ $[0: n]$ and $w \in \mathbb{W}^{n}$, since $\mu$ and $v$ are regular and $Q$ is bounded, the function

$$
f:\left[T_{k}, T_{k+1}\right] \times \mathbb{R}^{1 \times d} \rightarrow \mathbb{R}^{1 \times d}, \quad f(t, y) \triangleq y \cdot \widehat{Q}(t, w, \mu(t, w), v(t, w))
$$

is measurable in the first and Lipschitz continuous in the second argument. Thus, using that $\mu, v$ and $\widehat{Q}$ are non-anticipative, a classical result, see [36, Theorem I.5.3], implies that for each initial condition $y \in \mathbb{R}^{1 \times d}$ there exists a unique Carathéodory solution $\varphi_{k}^{y, w_{T_{k}}}:\left[T_{k}, T_{k+1}\right\rangle \rightarrow \mathbb{R}^{1 \times d}$ of

$$
\dot{y}(t)=y(t) \cdot \widehat{Q}\left(t, w_{T_{k}}, \mu\left(t, w_{T_{k}}\right), v\left(t, w_{T_{k}}\right)\right) \text { for } t \in\left[T_{k}, T_{k+1}\right\rangle, \quad y\left(T_{k}\right)=y .
$$

Step 2: $Y$ is $\mathfrak{G}$-adapted. First note that $Y_{0}=m_{0}$ is clearly $\mathfrak{G}_{0}$-measurable. Next, suppose that $Y_{T_{k}}$ is $\mathfrak{G}_{T_{k}}$-measurable, and note that for $t \in\left[T_{k}, T_{k+1}\right\rangle$ we have $W_{t}=$ $W_{T_{k}}$, so

$$
Y_{t}=Y_{T_{k}}+\int_{T_{k}}^{t} Y_{s} \cdot \widehat{Q}\left(s, W_{T_{k}}, \mu\left(s, W_{T_{k}}\right), v\left(s, W_{T_{k}}\right)\right) \mathrm{d} s .
$$

Thus from uniqueness in part (a) it follows that we have the representation

$$
Y_{t}=\varphi_{k}^{Y_{T_{k}}, W_{T_{k}}}(t) \text { for } t \in\left[T_{k}, T_{k+1}\right\rangle .
$$

Hence $Y_{t}$ is $\mathfrak{G}_{T_{k}}$-measurable for all $t \in\left[T_{k}, T_{k+1}\right\rangle$. Finally, for all $k \in[0:(n-1)]$ the consistency condition implies that $Y_{T_{k+1}}=\Phi_{k+1}\left(W_{T_{k+1}}, Y_{T_{k+1}-}, \mu\left(T_{k+1}-, W_{T_{k+1}-}\right)\right)$ is $\mathfrak{G}_{T_{k+1}}$-measurable, so the claim follows by induction on $k \in[0: n]$.

Theorem 9 (Aggregation) Let $\mu:[0, T] \times \mathbb{W}^{n} \rightarrow \mathbb{M}$ be regular and non-anticipative with $\mu(0)=m_{0}$. Suppose $v$ is a solution of $\left(D P_{\mu}\right)$ subject to $\left(C C_{\mu}\right),\left(T C_{\mu}\right)$, and the agent implements his optimal strategy $\widehat{v}$ as defined in Theorem 6 . Then the aggregate distribution $\widehat{M}$ has the $\widehat{\mathbb{P}}$-dynamics

$$
\mathrm{d} \widehat{M}_{t}=\widehat{M}_{t} \cdot \widehat{Q}\left(t, W_{t}, \mu\left(t, W_{t}\right), v\left(t, W_{t}\right)\right) \mathrm{d} t \text { for } t \in\left[T_{k}, T_{k+1}\right\rangle, k \in[0: n],
$$


and satisfies the initial condition

$$
\widehat{M}_{0}=m_{0}
$$

and the jump conditions

$$
\widehat{M}_{T_{k}}=\Phi_{k}\left(W_{T_{k}}, \widehat{M}_{T_{k}-}, \mu\left(T_{k}-, W_{T_{k}-}\right)\right) \text { for } k \in[1: n] .
$$

Proof Let $w \in \mathbb{W}^{n}$ be a common noise configuration. Since $X$ is defined path by path, see (2) and (3), we first note that $X=X^{w}$ on $\left\{W_{T}=w\right\}$, where $X^{w}$ satisfies (2) and

$$
X_{T_{k}}^{w}=J^{X_{T_{k}-}^{w}}\left(T_{k}, w_{T_{k}}, \mu\left(T_{k}-, w_{T_{k}-}\right)\right) \text { for } k \in[1: n] .
$$

We define $\zeta(w)=\left\{\zeta(w)_{t}\right\}$ via

$$
\begin{aligned}
\zeta(w)_{t} \triangleq & \prod_{\substack{i, j \in \mathbb{S}, i \neq j}}\left(\exp \left\{\int_{0}^{t}\left(1-Q^{i j}\left(s, w_{s}, \mu\left(s, w_{s}\right), h^{X_{s-}^{w}}\left(s, w_{s}, \mu\left(s, w_{s}\right), v\left(s, w_{s}\right)\right)\right)\right) \mathrm{d} s\right\}\right. \\
& \left.\times \prod_{\substack{s \in(0, t], \Delta N_{s}^{i j} \neq 0}} Q^{i j}\left(s, w_{s}, \mu\left(s, w_{s}\right), h^{X_{s-}^{w}}\left(s, w_{s}, \mu\left(s, w_{s}\right), v\left(s, w_{s}\right)\right)\right)\right) .
\end{aligned}
$$

Using analogous arguments as in Step 1 of the proof of Lemma 2 (see in particular (4) and (7)), it follows that there exists a probability measure $\widehat{\mathbb{P}}^{w}$ with density process

$$
\left.\frac{\mathrm{d} \widehat{\mathbb{P}}^{w}}{\mathrm{~d} \mathbb{P}}\right|_{\mathfrak{H}_{t}} \triangleq \zeta(w)_{t} \quad \text { for } t \in[0, T]
$$

where the filtration $\mathfrak{H}=\left\{\mathfrak{H}_{t}\right\}$ is given by

$$
\mathfrak{H}_{t} \triangleq \sigma\left(X_{0}, N_{S}^{i j}: s \in[0, t] ; i, j \in \mathbb{S}, i \neq j\right) \vee \mathfrak{N} \text { for } t \in[0, T]
$$

Furthermore, in view of (4) and (15) we have

$$
\zeta(w)=\mathcal{E}\left[\theta^{\widehat{v}}\right] \text { on }\left\{W_{T}=w\right\} .
$$

Step 1: Conditional Kolmogorov dynamics. Throughout Step 1, we fix a common noise configuration $w \in \mathbb{W}^{n}$. It follows exactly as in the proof of Lemma 2 (with $\widehat{\mathbb{P}} w$ in place of $\widehat{\mathbb{P}}$ ) that

$$
\widehat{\mathbb{P}}^{w} \ll \mathbb{P}, \quad \widehat{\mathbb{P}}^{w}=\mathbb{P} \text { on } \sigma\left(X_{0}\right),
$$

and that for $i, j \in \mathbb{S}, i \neq j$, the process $N^{i j}$ is a counting process with $\left(\mathfrak{H}, \widehat{\mathbb{P}}^{w}\right)$ intensity

$$
Q^{i j}\left(t, w_{t}, \mu\left(t, w_{t}\right), h^{X_{t-}^{w}}\left(t, w_{t}, \mu\left(t, w_{t}\right), v\left(t, w_{t}\right)\right)\right) \text { for } t \in[0, T]
$$


Boundedness of $Q$ implies that for each $z \in \mathbb{R}^{d}$ the process $L^{w}[z]=\left\{L_{t}^{w}[z]\right\}$,

$$
L_{t}^{w}[z] \triangleq \sum_{\substack{i, j \in \mathbb{S}, i \neq j}} \int_{0}^{t} \mathbb{1}_{\left\{X_{s-}^{w}=i\right\}} \cdot\left(z^{j}-z^{i}\right) \mathrm{d}^{w} \bar{N}_{s}^{i j} \quad \text { for } t \in[0, T]
$$

is an $\left(\mathfrak{H}, \widehat{\mathbb{P}}^{w}\right)$-martingale, where ${ }^{w} \bar{N}^{i j}=\left\{{ }^{w} \bar{N}_{t}^{i j}\right\}$ is given by

$$
{ }^{w} \bar{N}_{t}^{i j} \triangleq N_{t}^{i j}-\int_{0}^{t} Q^{i j}\left(s, w_{s}, \mu\left(s, w_{s}\right), h^{X_{s-}^{w}}\left(s, w_{s}, \mu\left(s, w_{s}\right), v\left(s, w_{s}\right)\right)\right) \mathrm{d} s, \quad t \in[0, T] .
$$

Using Itô's lemma and the fact that $\widehat{\lambda}_{t}^{i j}=\widehat{Q}^{i j}\left(t, W_{t}, \mu\left(t, W_{t}\right), v\left(t, W_{t}\right)\right)$ on $\left\{X_{t-}=\right.$ $i\}, t \in[0, T]$, by (15), we have for each $z \in \mathbb{R}^{d}, k \in[0: n]$ and $t \in\left[T_{k}, T_{k+1}\right\rangle$

$$
z^{X_{t}^{w}}=z^{X_{T_{k}}^{w}}+L_{t}^{w}[z]-L_{T_{k}}^{w}[z]+\sum_{i=1}^{d} \int_{T_{k}}^{t} \mathbb{1}_{\left\{X_{s}^{w}=i\right\}} \cdot \widehat{Q}^{i \cdot}\left(s, w_{s}, \mu\left(s, w_{s}\right), v\left(s, w_{s}\right)\right) \cdot z \mathrm{~d} s
$$

Taking expectations with respect to $\widehat{\mathbb{P}}^{w}$ and using Fubini's theorem yields

$\widehat{\mathbb{E}}^{w}\left[z^{X_{t}^{w}}\right]=\widehat{\mathbb{E}}^{w}\left[z^{X_{T_{k}}^{w}}\right]+\sum_{i=1}^{d} \int_{T_{k}}^{t} \widehat{\mathbb{P}}^{w}\left(X_{s}^{w}=i\right) \cdot \widehat{Q}^{i \cdot}\left(s, w_{s}, \mu\left(s, w_{s}\right), v\left(s, w_{s}\right)\right) \cdot z \mathrm{~d} s$,

so with $z=e_{i}, i \in \mathbb{S}$, we get

$$
\widehat{\mathbb{P}}^{w}\left(X_{t}^{w}=i\right)=\widehat{\mathbb{P}}^{w}\left(X_{T_{k}}^{w}=i\right)+\sum_{j=1}^{d} \int_{T_{k}}^{t} \widehat{\mathbb{P}}^{w}\left(X_{s}^{w}=j\right) \cdot \widehat{Q}^{j i}\left(s, w_{s}, \mu\left(s, w_{s}\right), v\left(s, w_{s}\right)\right) \mathrm{d} s .
$$

It follows from (20) that $\eta(w)=\left\{\eta(w)_{t}\right\}$,

$$
\eta(w)_{t} \triangleq \widehat{\mathbb{P}}^{w}\left(X_{t}^{w} \in \cdot\right), \quad t \in[0, T]
$$

satisfies, for all $i \in \mathbb{S}$ and $k \in[0: n]$,

$$
\eta(w)_{t}^{i}=\eta(w)_{T_{k}}^{i}+\int_{T_{k}}^{t} \eta(w)_{s} \cdot \widehat{Q}^{i}\left(s, w_{s}, \mu\left(s, w_{s}\right), v\left(s, w_{s}\right)\right) \mathrm{d} s \quad \text { for } t \in\left[T_{k}, T_{k+1}\right\rangle .
$$

Moreover, since $\widehat{\mathbb{P}}^{w}=\mathbb{P}$ on $\sigma\left(X_{0}\right)$ and $X_{0}^{w}=X_{0}, \eta(w)$ satisfies the initial condition

$$
\eta(w)_{0}=\widehat{\mathbb{P}}^{w}\left(X_{0}^{w} \in \cdot\right)=\mathbb{P}\left(X_{0}^{w} \in \cdot\right)=\mathbb{P}\left(X_{0} \in \cdot\right)=m_{0}
$$


Finally, consider a common noise time $t=T_{k}$ and note that for all $i \in \mathbb{S}$ the jump condition (18) implies

$$
\begin{aligned}
\eta(w)_{T_{k}}^{i} & =\widehat{\mathbb{P}}^{w}\left(X_{T_{k}}^{w}=i\right)=\widehat{\mathbb{P}}^{w}\left(J^{X_{T_{k}}}{ }^{w}\left(T_{k}, w_{T_{k}}, \mu\left(T_{k}-, w_{T_{k}-}\right)\right)=i\right) \\
& =\sum_{j=1}^{d} \widehat{\mathbb{P}}^{w}\left(J^{j}\left(T_{k}, w_{T_{k}}, \mu\left(T_{k}-, w_{T_{k}-}\right)\right)=i \mid X_{T_{k}-}^{w}=j\right) \cdot \widehat{\mathbb{P}}^{w}\left(X_{T_{k}-}^{w}=j\right) \\
& =\sum_{j=1}^{d} \mathbb{1}_{\left\{J^{j}\left(T_{k}, w_{T_{k}}, \mu\left(T_{k}-, w_{T_{k}-}\right)\right)=i\right\}} \cdot \widehat{\mathbb{P}}^{w}\left(X_{T_{k}-}^{w}=j\right) \\
& =\sum_{j=1}^{d} P_{k}^{j i}\left(w_{T_{k}}, \mu\left(T_{k}-, w_{T_{k}-}\right)\right) \cdot \eta(w)_{T_{k}-}^{j}=\Phi_{k}^{i}\left(w_{T_{k}}, \eta(w)_{T_{k}-}, \mu\left(T_{k}-, w_{T_{k}-}\right)\right) .
\end{aligned}
$$

Since $\eta\left(W_{T}\right)=\sum_{w \in \mathbb{W}^{n}} \mathbb{1}_{\left\{W_{T}=w\right\}} \cdot \eta(w)$, in view of (22), (23) and (24) it follows from Lemma 8 that the process $\eta\left(W_{T}\right)$ is $\mathfrak{G}$-adapted.

Step 2: Identification of $\eta\left(W_{T}\right)$. Recall that $\mathfrak{G}_{T}=\sigma\left(W_{T}\right) \vee \mathfrak{N}$ and let $w \in \mathbb{W}^{n}$. For $t \in[0, T]$ and $i \in \mathbb{S}$ we have by (6) and (19)

$$
\begin{aligned}
& \widehat{\mathbb{E}}\left[\mathbb{1}_{\left\{W_{T}=w\right\}} \cdot \mathbb{1}_{\left\{X_{t}=i\right\}}\right]=\mathbb{E}\left[\mathbb{1}_{\left\{W_{T}=w\right\}} \cdot \mathbb{1}_{\left\{X_{t}^{w}=i\right\}} \cdot Z_{T}^{\widehat{v}}\right]=\mathbb{E}\left[\mathbb{1}_{\left\{W_{T}=w\right\}} \cdot \mathbb{1}_{\left\{X_{t}^{w}=i\right\}} \cdot \zeta(w)_{T} \cdot \mathcal{E}[\vartheta]_{T}\right] \\
& =\prod_{k=1}^{n}\left(|\mathbb{W}| \cdot \kappa_{k}\left(w_{k} \mid w_{1}, \ldots, w_{k-1}, \mu\left(T_{k}-, w_{T_{k}-}\right)\right)\right) \cdot \mathbb{E}\left[\mathbb{1}_{\left\{W_{T}=w\right\}} \cdot \mathbb{1}_{\left\{X_{t}^{w}=i\right\}} \cdot \zeta(w)_{T}\right] \\
& =|\mathbb{W}|^{n} \cdot \widehat{\mathbb{P}}\left(W_{T}=w\right) \cdot \mathbb{P}\left(W_{T}=w\right) \cdot \widehat{\mathbb{P}}^{w}\left(X_{t}^{w}=i\right)=\widehat{\mathbb{E}}\left[\mathbb{1}_{\left\{W_{T}=w\right\}} \cdot \eta\left(W_{T}\right)_{t}^{i}\right],
\end{aligned}
$$

where in the final line the first identity is due to Lemma 2 and $\mathbb{P}$-independence of $\left(\zeta(w), X^{w}\right)$ and $\mathfrak{G}_{T}$; and the second is due to (21) and the fact that $\mathbb{P}\left(W_{T}=w\right)=$ $1 /|\mathbb{W}|^{n}$. Thus

$$
\widehat{\mathbb{P}}\left(X_{t} \in \cdot \mid \mathfrak{G}_{T}\right)=\eta\left(W_{T}\right)_{t} \widehat{\mathbb{P}} \text {-a.s. for } t \in[0, T] .
$$

Step 3: Dynamics of $\widehat{M}$. By Step 2 and the tower property of conditional expectation, we find that for each $i \in \mathbb{S}$ and $t \in[0, T]$

$\widehat{M}_{t}^{i}=\widehat{\mathbb{P}}\left(X_{t}=i \mid \mathfrak{G}_{t}\right)=\widehat{\mathbb{E}}\left[\widehat{\mathbb{E}}\left[\mathbb{1}_{\left\{X_{t}=i\right\}} \mid \mathfrak{G}_{T}\right] \mid \mathfrak{G}_{t}\right]=\widehat{\mathbb{E}}\left[\eta\left(W_{T}\right)_{t}^{i} \mid \mathfrak{G}_{t}\right]=\eta\left(W_{T}\right)_{t}^{i} \quad \widehat{\mathbb{P}}$-a.s.

where the final identity is due to the fact that $\eta\left(W_{T}\right)$ is $\mathfrak{G}$-adapted by Step 1 and $\widehat{\mathbb{E}}$ denotes $\widehat{\mathbb{P}}$-expectation. Since both $\widehat{M}$ and $\eta\left(W_{T}\right)$ are càdlàg, it follows that $\widehat{M}=$ $\eta\left(W_{T}\right) \widehat{\mathbb{P}}$-a.s., and $(\mathrm{M}),\left(\mathrm{M}_{0}\right)$ and $\left(\mathrm{M}_{k}\right)$ follow from (22), (23) and (24).

As a by-product, the preceding proof yields the alternative representation

$$
\widehat{M}_{t}=\widehat{\mathbb{P}}\left(X_{t} \in \cdot \mid \mathfrak{G}_{T}\right) \text { for } t \in[0, T], \widehat{\mathbb{P}} \text {-a.s. }
$$




\subsection{Mean Field Equilibrium System}

As discussed above, equilibrium obtains if the agents' ex ante beliefs coincide with the ex post outcome. This holds if and only if the ex post aggregate distribution process $\widehat{M}$ from (M) satisfies

$$
\widehat{\mathbb{P}}\left(X_{t} \in \cdot \mid \mathfrak{G}_{t}\right)=\widehat{M}_{t} \stackrel{!}{=} M_{t}=\mu\left(t, W_{t}\right) \text { for all } t \in[0, T] .
$$

Definition 10 (Equilibrium System). A pair $(\mu, v)$ of regular and non-anticipative functions

$$
\mu:[0, T] \times \mathbb{W}^{n} \rightarrow \mathbb{M} \quad \text { and } \quad v:[0, T] \times \mathbb{W}^{n} \rightarrow \mathbb{R}^{d}
$$

is called a rational expectations equilibrium, or briefly an equilibrium, if for all $w \in$ $\mathbb{W}^{n}$

$$
\begin{gathered}
\dot{\mu}(t, w)=\mu(t, w) \cdot \widehat{Q}(t, w, \mu(t, w), v(t, w)) \\
\dot{v}(t, w)=-\widehat{\psi}(t, w, \mu(t, w), v(t, w))-\widehat{Q}(t, w, \mu(t, w), v(t, w)) \cdot v(t, w)
\end{gathered}
$$

for $t \in\left[T_{k}, T_{k+1}\right\rangle, k \in[0: n]$, subject to the consistency conditions ${ }^{7}$

$$
\begin{gathered}
\mu\left(T_{k}, w\right)=\Phi_{k}\left(w, \mu\left(T_{k}-, w\right)\right) \\
v\left(T_{k}-, w\right)=\Psi_{k}\left(w, \mu\left(T_{k}-, w\right), v\left(T_{k}, \cdot\right)\right)
\end{gathered}
$$

for $k \in[1: n]$, and the initial/terminal conditions

$$
\begin{gathered}
\mu(0, w)=m_{0} \\
v(T, w)=\Psi(w, \mu(T, w)) .
\end{gathered}
$$

We also refer to (E1)-(E6) as the equilibrium system.

In combination, Theorem 6 and Theorem 9 demonstrate that, given a solution $(\mu, v)$ of the equilibrium system, $v$ is the value function of the agent's optimization problem $\left(\mathrm{P}_{\mu}\right)$ with ex ante aggregate distribution $\mu$; and the ex post distribution resulting from the corresponding optimal strategy is given by $\mu$ itself. Thus we can identify a mean field equilibrium with common noise by producing a solution of the equilibrium system (E1)-(E6). We provide some illustrations in Sect. 5. Theorems 13 and 16 below ensure that this is feasible by showing that, under suitable continuity and monotonicity conditions, there exists a unique solution of the equilibrium system. The proofs are

\footnotetext{
7 With a slight abuse of notation, here and subsequently we set $\Phi_{k}(w, m) \triangleq \Phi_{k}(w, m, m)$ for $k \in[1: n]$, $w \in \mathbb{W}^{n}, m \in \mathbb{M}$. Recall that $\Psi_{k}$ and $\Phi_{k}$ are defined in (10) and (16), respectively.
} 
ramifications of classical arguments, based on Schauder's fixed point theorem and monotonicity arguments, respectively.

We set

$$
\begin{aligned}
& Q_{\max } \triangleq \sup _{\substack{t \in[0, T], w \in \mathbb{W}^{n} \\
m \in \mathbb{M}, u \in \mathbb{U}}}\|Q(t, w, m, u)\|, \quad \psi_{\max } \triangleq \sup _{\substack{t \in[0, T], w \in \mathbb{W}^{n} \\
m \in \mathbb{M}, u \in \mathbb{U}}}\|\psi(t, w, m, u)\|, \\
& \Psi_{\max } \triangleq \sup _{\substack{m \in \mathbb{M} \\
w \in \mathbb{W}^{n}}}\|\Psi(w, m)\|
\end{aligned}
$$

and

$$
v_{\max } \triangleq\left(\Psi_{\max }+T \cdot \psi_{\max }\right) \cdot \mathrm{e}^{Q_{\max } \cdot T}
$$

Note that these constants depend only on the underlying model coefficients.

Assumption 11 (i) The reduced-form running reward function $\widehat{\psi}$ satisfies

$$
\left\|\widehat{\psi}\left(t, w, m_{1}, v_{1}\right)-\widehat{\psi}\left(t, w, m_{2}, v_{2}\right)\right\| \leq L_{\widehat{\psi}} \cdot\left(\left\|m_{1}-m_{2}\right\|+\left\|v_{1}-v_{2}\right\|\right)
$$

for all $t \in[0, T], w \in \mathbb{W}^{n}, m_{1}, m_{2} \in \mathbb{M}$ and $v_{1}, v_{2} \in \mathbb{R}^{d}$ with $\left\|v_{1}\right\|,\left\|v_{2}\right\| \leq$ $v_{\max }$, for some $L_{\widehat{\psi}}>0$.

(ii) The reduced-form intensity matrix function $\widehat{Q}$ satisfies

$$
\left\|\widehat{Q}\left(t, w, m_{1}, v_{1}\right)-\widehat{Q}\left(t, w, m_{2}, v_{2}\right)\right\| \leq L_{\widehat{Q}} \cdot\left(\left\|m_{1}-m_{2}\right\|+\left\|v_{1}-v_{2}\right\|\right)
$$

for all $t \in[0, T], w \in \mathbb{W}^{n}, m_{1}, m_{2} \in \mathbb{M}$ and $v_{1}, v_{2} \in \mathbb{R}^{d}$ with $\left\|v_{1}\right\|,\left\|v_{2}\right\| \leq$ $v_{\max }$, for some $L_{\widehat{Q}}>0$.

(iii) The terminal reward function $\Psi$ is continuous with respect to $m$, i.e. for every $w \in \mathbb{W}^{n}$ the map $\Psi(w, \cdot)$ is continuous.

(iv) For each $k \in[1: n]$ and all $i \in \mathbb{S}, w \in \mathbb{W}^{n}$ and $v \in \mathbb{R}^{d}$ with $\|v\| \leq v_{\max }$, the map

$$
\mathbb{M} \ni m \mapsto \sum_{\bar{w}_{k} \in \mathbb{W}} \kappa_{k}\left(\bar{w}_{k} \mid w_{1}, \ldots, w_{k-1}, m\right) v^{J^{i}\left(T_{k},\left(w_{-k}, \bar{w}_{k}\right), m\right)} \in \mathbb{R} \quad \text { is continuous. }
$$

(v) For each $k \in[1: n]$ and $w \in \mathbb{W}^{n}$ the map $\Phi_{k}(w, \cdot)$ is continuous.

Since all norms on $\mathbb{R}^{d}$ are equivalent, the concrete specification is immaterial for Assumption 11. For the sake of convenience, in the following we use the maximum norm on $\mathbb{R}^{d}$ and a compatible matrix norm on $\mathbb{R}^{d \times d}$; moreover, we suppose that (ii) holds for both $\widehat{Q}$ and $\widehat{Q}^{\top}$.

Remark 12 Sufficient conditions for Assumptions 11(i)-(ii) in terms of the model's primitives can be found in, e.g., [31] or [15]. Furthermore, in the special case where the jump map $J$ is independent of $m \in \mathbb{M}$, Assumption $11(\mathrm{v})$ is trivially satisfied, 
and continuity of the transition kernels $\kappa_{k}$ with respect to $m$ is sufficient for Assumption 11(iv) to hold.

Theorem 13 (Existence of Equilibria) If Assumption 11 holds, then there exists a solution of the equilibrium system (E1)-(E6).

Proof See Appendix A.

The reduced-form Hamiltonian $\widehat{\mathcal{H}}:[0, T] \times \mathbb{W}^{n} \times \mathbb{M} \times \mathbb{R}^{d} \rightarrow \mathbb{R}^{d}$ is defined via

$$
\begin{aligned}
\widehat{\mathcal{H}}^{i}(t, w, m, v) & \triangleq \sup _{u \in \mathbb{U}} \psi^{i}(t, w, m, u)+Q^{i \cdot}(t, w, m, u) \cdot v \\
& =\widehat{\psi}^{i}(t, w, m, v)+\widehat{Q}^{i \cdot}(t, w, m, v) \cdot v .
\end{aligned}
$$

Assumption 14 Let Assumptions 11(i) and (ii) hold, and suppose that:

(i) The terminal payoff function $\Psi$ is monotone with respect to $m \in \mathbb{M}$, i.e.

$$
\left(m_{1}-m_{2}\right) \cdot\left[\Psi\left(w, m_{1}\right)-\Psi\left(w, m_{2}\right)\right] \leq 0 \text { for all } w \in \mathbb{W}^{n}, m_{1}, m_{2} \in \mathbb{M}
$$

(ii) The reduced-form Hamiltonian $\widehat{\mathcal{H}}$ is convex with respect to $v$, i.e. for all $i \in \mathbb{S}$, $t \in[0, T], w \in \mathbb{W}^{n}, m \in \mathbb{M}$ and $v_{1}, v_{2} \in \mathbb{R}^{d}$ satisfying $\left\|v_{1}\right\|,\left\|v_{2}\right\| \leq v_{\max }$ we have

$$
\widehat{\mathcal{H}}^{i}\left(t, w, m, v_{2}\right)-\widehat{\mathcal{H}}^{i}\left(t, w, m, v_{1}\right)-\widehat{Q}^{i \cdot}\left(t, w, m, v_{1}\right) \cdot\left(v_{2}-v_{1}\right) \geq 0
$$

(iii) The reduced-form Hamiltonian $\widehat{\mathcal{H}}$ satisfies a uniform monotonicity condition with respect to $m \in \mathbb{M}$, i.e. there exist $\alpha, \gamma>0$ such that

$$
\begin{aligned}
m_{1} \cdot\left[\widehat{\mathcal{H}}\left(t, w, m_{2}, v_{2}\right)-\widehat{\mathcal{H}}\left(t, w, m_{1}, v_{2}\right)\right]+m_{2} \cdot & {\left[\widehat{\mathcal{H}}\left(t, w, m_{1}, v_{1}\right)\right.} \\
& \left.-\widehat{\mathcal{H}}\left(t, w, m_{2}, v_{1}\right)\right] \geq \gamma \cdot\left\|m_{1}-m_{2}\right\|^{\alpha}
\end{aligned}
$$

for all $t \in[0, T], w \in \mathbb{W}^{n}, m_{1}, m_{2} \in \mathbb{M}$ and $v_{1}, v_{2} \in \mathbb{R}^{d}$ with $\left\|v_{1}\right\|,\left\|v_{2}\right\| \leq$ $v_{\text {max }}$.

(iv) For $k \in[1: n]$ the maps $\kappa_{k}$ and $J$ satisfy the following monotonicity conditions in $m \in \mathbb{M}$ : For all $w \in \mathbb{W}^{n}, m_{1}, m_{2} \in \mathbb{M}$ and $v_{1}, v_{2} \in \mathbb{R}^{d}$ satisfying $\left\|v_{1}\right\|,\left\|v_{2}\right\| \leq$ $v_{\max }$ as well as

$$
\left[\Phi_{k}\left(w, m_{1}\right)-\Phi_{k}\left(w, m_{2}\right)\right] \cdot\left(v_{1}-v_{2}\right) \leq 0
$$

we have

$$
\begin{aligned}
& {\left[\kappa_{k}\left(w_{k} \mid w_{1}, \ldots, w_{k-1}, m_{1}\right)-\kappa_{k}\left(w_{k} \mid w_{1}, \ldots, w_{k-1}, m_{2}\right)\right]} \\
& \quad \cdot\left(m_{2} \cdot v_{1}^{J \cdot\left(T_{k}, w, m_{2}\right)}-m_{1} \cdot v_{1}^{J \cdot\left(T_{k}, w, m_{1}\right)}\right. \\
& \left.\quad+m_{2} \cdot v_{2}^{J \cdot\left(T_{k}, w, m_{2}\right)}-m_{1} \cdot v_{2}^{J \cdot\left(T_{k}, w, m_{1}\right)}\right) \geq 0
\end{aligned}
$$


and

$$
\begin{aligned}
& \kappa_{k}\left(w_{k} \mid w_{1}, \ldots, w_{k-1}, m_{2}\right) \cdot m_{1} \cdot\left(v_{2}^{J \cdot\left(T_{k}, w, m_{2}\right)}-v_{2}^{J \cdot\left(T_{k}, w, m_{1}\right)}\right) \\
& \quad+\kappa_{k}\left(w_{k} \mid w_{1}, \ldots, w_{k-1}, m_{1}\right) \cdot m_{2} \cdot\left(v_{1}^{J \cdot\left(T_{k}, w, m_{1}\right)}-v_{1}^{J \cdot\left(T_{k}, w, m_{2}\right)}\right) \geq 0 .
\end{aligned}
$$

The constant $v_{\max }>0$ in 14(ii)-(iv) is defined in (25). Conditions 14(i)-(iii) are standard given the literature; see, e.g., Assumptions 1-3 in [31]. ${ }^{8}$

Remark 15 Assumption 14 simplifies if some model coefficients do not depend on the mean field parameter $m \in \mathbb{M}$ :

(a) If $\widehat{Q}$ is independent of $m, 14$ (iii) reduces to a monotonicity condition for $\widehat{\psi}$.

(b) In 14(iv), (26) is trivially satisfied if the probability weights $\kappa_{k}$ do not depend on $m$.

(c) In 14(iv), (27) is trivially satisfied if the jump map $J$ is independent of $m$.

Theorem 16 (Uniqueness of Equilibria) Under the monoticity conditions stated in Assumption 14, the equilibrium system (E1)-(E6) possesses at most one solution.

Proof See Appendix B.

\section{Applications}

Before we illustrate our results in two showcase examples, we briefly discuss our numerical approach to the equilibrium system (E1)-(E6). (E1)-(E2) is a forwardbackward system of $2 d$ ODEs with boundary conditions (E3)-(E6), coupled through the parameter $w \in \mathbb{W}^{n}$ representing common noise configurations. The special case $n=0$ (no common noise) corresponds to the setting of [31] and [15], with the equilibrium system reducing to a single $2 d$-dimensional forward-backward ODE. For $n \geq 1$, the consistency conditions (E3)-(E4) specify initial conditions for $\mu$ on $\left[T_{k}, T_{k+1}\right\rangle$ and terminal conditions for $v$ on $\left[T_{k-1}, T_{k}\right\rangle, k \in[1: n]$; since these conditions are interconnected, there is in general no segment $\left[T_{k}, T_{k+1}\right\rangle \times \mathbb{W}^{n}$ where the equilibrium system yields both an explicit initial condition for $\mu$ and an explicit terminal condition for $v$, so we cannot simply split the problem into subintervals. Rather, the equilibrium system can be regarded as a multi-point boundary value problem where for each of the $|\mathbb{W}|^{k}$ conceivable combinations of common noise factors on $\left[T_{k}, T_{k+1}\right\rangle, k \in[0: n]$, we have to solve a coupled forward-backward system of ODEs in $2 d$ dimensions, resulting in a tree of such systems of size

$$
\sum_{k=0}^{n}|\mathbb{W}|^{k}=\frac{|\mathbb{W}|^{n+1}-1}{|\mathbb{W}|-1} \in \mathcal{O}\left(\left|\mathbb{W}^{n}\right|\right)
$$

Our approach to solving (E1)-(E6) numerically is to rely on the probabilistic interpretation as a fixed-point system, based on Theorem 13. Thus, starting from an initial

\footnotetext{
8 Note, however, that our result does not require uniform convexity in 14(ii).
} 
flow of probability weights $\mu_{0}(t, w),(t, w) \in[0, T] \times \mathbb{W}^{n}$ with $\mu_{0}(0, w)=m_{0}$ for all $w \in \mathbb{W}^{n}$, we solve $\left(\mathrm{DP}_{\mu}\right)$ subject to $\left(\mathrm{TC}_{\mu}\right)$ and $\left(\mathrm{CC}_{\mu}\right)$ backward in time for all non-negligible common noise configurations $w \in \mathbb{W}^{n}$ to obtain the value $v_{0}(t, w)$, $(t, w) \in[0, T] \times \mathbb{W}^{n}$, of the agents' optimal response to the given belief $\mu_{0}$. This, in turn, is used to solve (M) subject to $\left(\mathrm{M}_{0}\right)$ and $\left(\mathrm{M}_{k}\right)$ forward in time. As a result, we obtain an ex post aggregate distribution $\mu_{1}(t, w),(t, w) \in[0, T] \times \mathbb{W}^{n}$; we then iterate this with $\mu_{1}$ in place of $\mu_{0}$, etc. ${ }^{9}$

\subsection{A Decentralized Agricultural Production Model}

As a first (stylized) example we consider a mean field game of agents, each of which owns (an infinitesimal amount of) land of identical size and quality within a given area. If it is farmed, each field has a productivity $f\left(w_{k}\right)>0$ depending on the common weather condition $w_{k}$. We assume that weather is either good, bad or catastrophic, so $w_{k} \in \mathbb{W} \triangleq\{\uparrow, \downarrow, \downarrow\}$, and changes at given common noise times $T_{1}, \ldots, T_{n}$.

Each agent is in exactly one state $i \in \mathbb{S} \triangleq\{0,1\}$ depending on whether he grows crops on his field ( $i=1$, the agent is a farmer) or not $(i=0)$. The selling price $p$ for his harvest depends on aggregate production, and thus in particular on the proportion $m^{1} \in[0,1]$ of farmers; the mean field interaction is transmitted through the market price of the crop. We assume that $p$ is a strictly decreasing function of overall production $f\left(w_{k}\right) \cdot m^{1}$; see Fig. 1 for illustration.

We assume that $f(\uparrow) \geq f(\downarrow)=f(\xi) \geq 0$. Moreover, on the catastrophic event $\left\{W_{k}=\{\}\right.$ all agents are reduced to being non-farmers, and thus

$$
J^{i}(t, w, m) \triangleq \begin{cases}0 & \text { if } t \in\left\{T_{1}, \ldots, T_{n}\right\}, t=T_{k}, w_{k}=\text { 々 } \\ i & \text { else }\end{cases}
$$

for $(i, t, w, m) \in \mathbb{S} \times[0, T] \times \mathbb{W}^{n} \times \mathbb{M}$. Each agent can make an effort $u \in \mathbb{U} \triangleq[0, \infty)$ to become being a farmer; the intensity matrix for state transitions is given by

$$
Q(t, w, m, u)=\left[\begin{array}{cc}
-u \cdot q_{\text {entry }} & u \cdot q_{\text {entry }} \\
q_{\text {exit }} & -q_{\text {exit }}
\end{array}\right] \text { for }(t, w, m, u) \in[0, T] \times \mathbb{W}^{n} \times \mathbb{M} \times \mathbb{U},
$$

where $q_{\text {entry }}, q_{\text {exit }} \geq 0$ are given transition rates. The running rewards capture the fact that both efforts to building up farming capacities and production itself are costly, while revenues from the sales of the crop generate profits; thus

$$
\psi^{0}(t, w, m, u)=-\frac{1}{2} c_{\text {entry }} \cdot u^{2} \quad \text { and } \quad \psi^{1}(t, w, m, u)=p\left(f\left(w_{k}\right) \cdot m^{1}\right) \cdot f\left(w_{k}\right)-c_{\text {prod }}
$$

for $t \in\left[T_{k}, T_{k+1}\right\rangle, k \in[0: n]$, where $w_{0} \triangleq \uparrow$ and $c_{\text {entry }}, c_{\text {prod }} \geq 0$. The terminal reward is zero. It follows that the maximizer $h^{0}$ in Assumption 3 is unique and given by

\footnotetext{
${ }^{9}$ Under suitable Lipschitz conditions, one can establish a variant of Theorem 13 based on Banach's (rather than Schauder's) fixed point theorem; see, e.g., [15, Theorem 6] for the case without common noise. This guarantees convergence of the fixed point iteration for sufficiently short time horizons.
} 


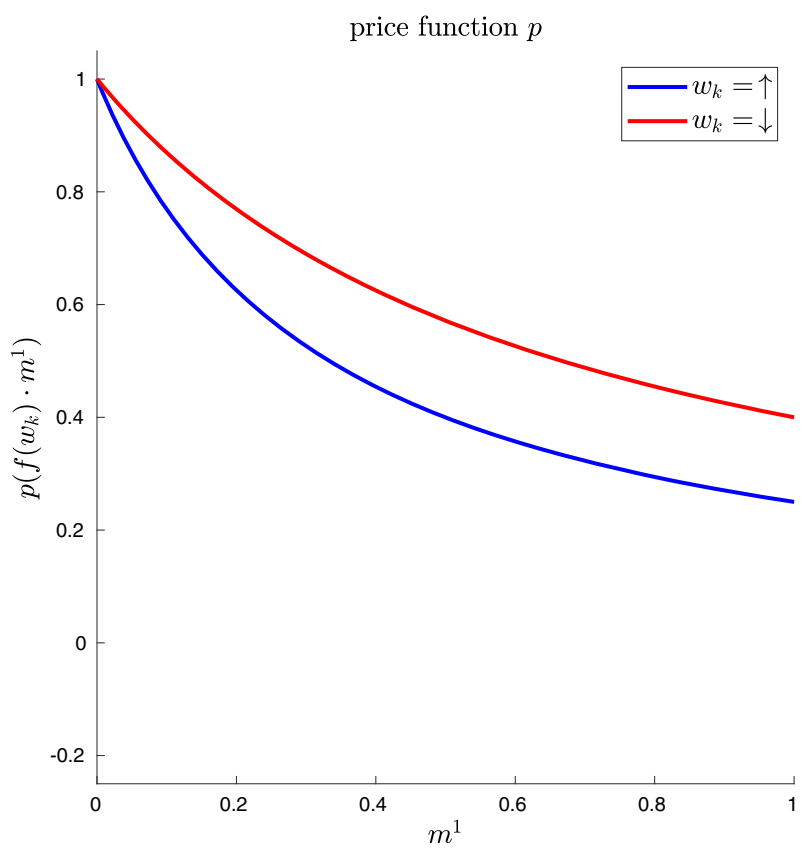

Fig. 1 Price function $p$ (parameters as in Table 1)

Table 1 Coefficients in the agricultural production model

\begin{tabular}{lcccccccccc}
\hline Parameter & $T$ & $n$ & $T_{k}$ & $q_{\text {entry }}$ & $q_{\text {exit }}$ & $f(\uparrow)$ & $f(\downarrow)$ & $p(q)$ & $c_{\text {prod }}$ & $c_{\text {entry }}$ \\
\hline Value & 1 & 4 & $k / 5$ & 1 & 0.1 & 1 & 0.5 & $1 /(1+3 q)$ & 0.3 & 0.1 \\
\hline
\end{tabular}

$$
h^{0}(t, w, m, v)=\frac{q_{\text {entry }}}{c_{\text {entry }}}\left(v^{1}-v^{0}\right)^{+}
$$

a specification of $h^{1}$ is immaterial. We choose $m_{0}^{1} \triangleq 10 \%$ for the initial proportion of farmers, and report the relevant coefficients in Table 1.

Our results for the evolution of the mean field equilibrium are shown in Figs. 2 and 3 for various common noise configurations $w \in \mathbb{W}^{n}$ and the following two baseline models:

(nC) Catastrophic weather conditions do not occur; we use

$$
\kappa_{k}\left(\uparrow \mid w_{1}, \ldots, w_{k-1}, m\right)=\kappa_{k}\left(\downarrow \mid w_{1}, \ldots, w_{k-1}, m\right)=0.5
$$

for all $w \in \mathbb{W}^{n}$ and $m \in \mathbb{M}$.

(C) Catastrophic events are likely; we use

$$
\begin{aligned}
& \kappa_{k}\left(\uparrow \mid w_{1}, \ldots, w_{k-1}, m\right)=0.25, \quad \kappa_{k}\left(\downarrow \mid w_{1}, \ldots, w_{k-1}, m\right)=0.25, \\
& \kappa_{k}\left(\downarrow \mid w_{1}, \ldots, w_{k-1}, m\right)=0.5
\end{aligned}
$$




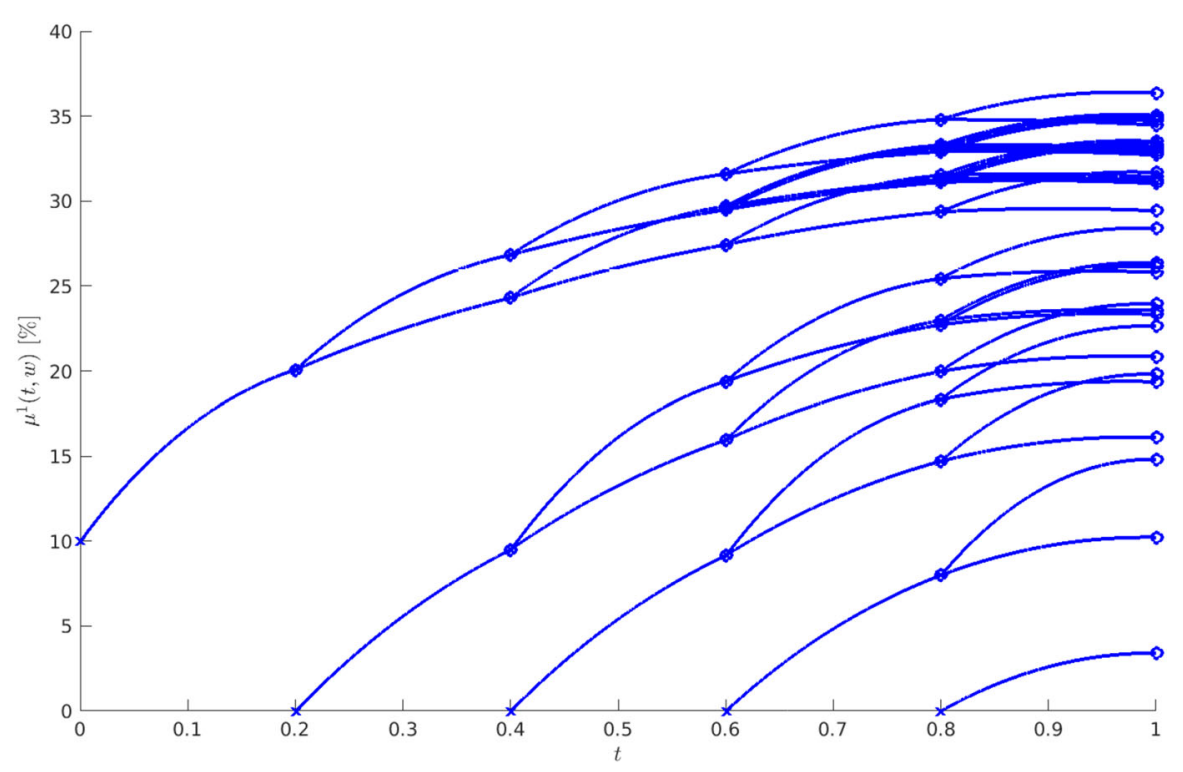

Fig. 2 Proportion of farmers in model (C) for all possible common noise configurations $w \in \mathbb{W}^{n}$

for all $w \in \mathbb{W}^{n}$ and $m \in \mathbb{M}$.

The model specified above satisfies both Assumption 11 and Assumption 14, so Theorems 13 and 16 guarantee the existence of a unique mean field equilibrium characterized by (E1)-(E6). Figure 2 illustrates the tree of all possible equilibrium evolutions in model (C). Figs. 3, 4 and 5 illustrate the resulting equilibrium proportions of farmers, optimal actions, and market prices for some fixed common noise configurations. To illustrate the effect of uncertainty about future weather conditions we also show, for each common noise configuration, the theoretical perfect-foresight equilibria that would pertain if future weather conditions were known; these are plotted using dashed lines in Figs. 3, 4 and 5, and the subscript $\mathrm{o}$ indicates the relevant deterministic common noise path. Equilibrium prices are stochastically modulated by the prevailing weather conditions, both directly and indirectly: First, prices jump at common noise times due to weather-related changes in productivity. Second, weather conditions indirectly affect market prices through their effect on the proportion of farming agents. Thus, with consistently good weather conditions, agents are strongly incentivized to become farmers, see Fig. 4; the fraction of farmers increases, see Fig. 3; and hence increased production drives down prices, see Fig. 5. By contrast, under bad weather conditions, incentives are weaker and prices remain higher. Both effects are dampened if a catastrophic event may occur. In addition, efforts tend to decrease between common noise times; this is due to the uncertainty of future weather conditions; this effect is more pronounced in the presence of catastrophic events. 
without catastrophe $(\mathrm{nC})$
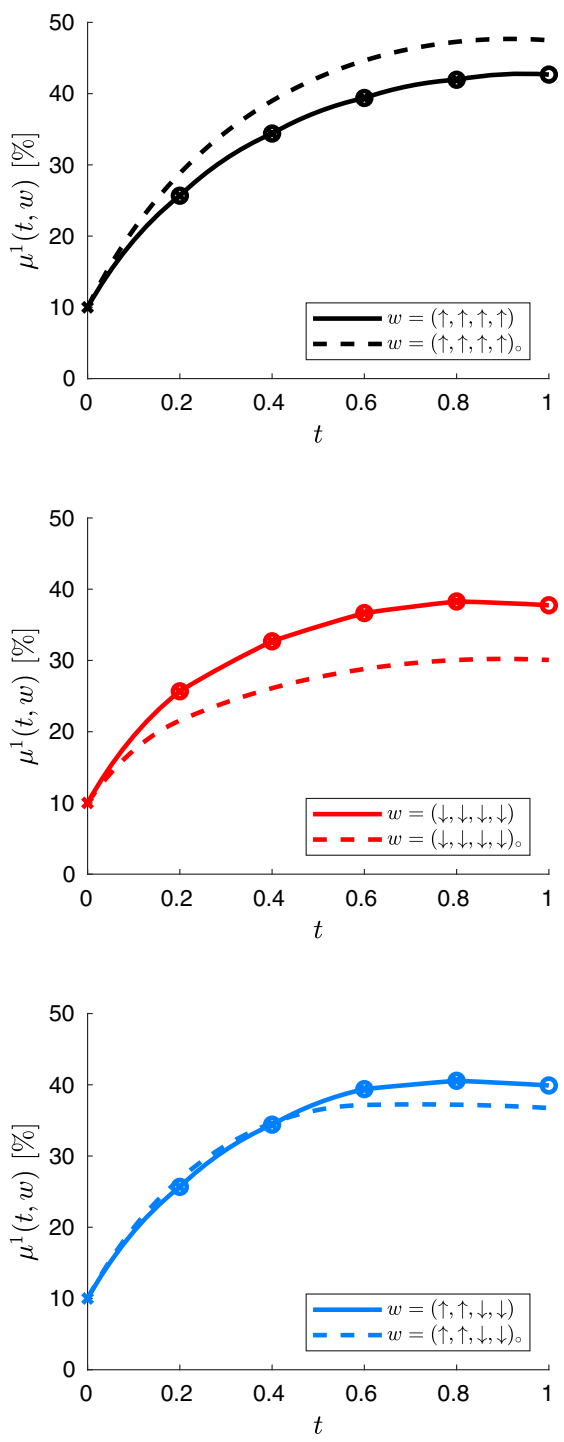

with catastrophe $(\mathrm{C})$
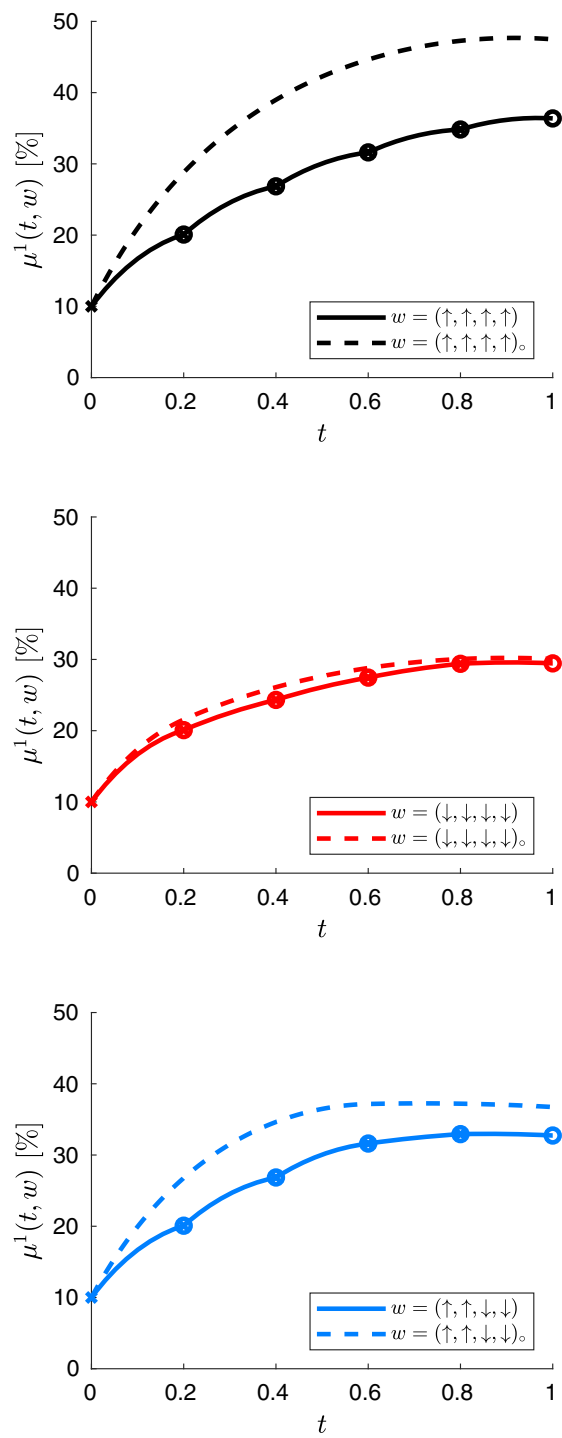

Fig. 3 Proportion of farmers in models (nC) and (C)

\subsection{An SIR Model with Random One-Shot Vaccination}

Our second application is a mean field game of agents that are confronted with the spread of an infectious disease. Our main focus is to illustrate the qualitative effects of common noise on the equilibrium behavior of the system. We consider a classical SIR model setup with $\mathbb{S}=\{\mathrm{S}, \mathrm{I}, \mathrm{R}\}$ : Each agent can be either susceptible to infection (S), infected and simultaneously infectious for other agents (I), or recovered and thus immune to (re-)infection (R); see Fig. 6. 
without catastrophe $(\mathrm{nC})$
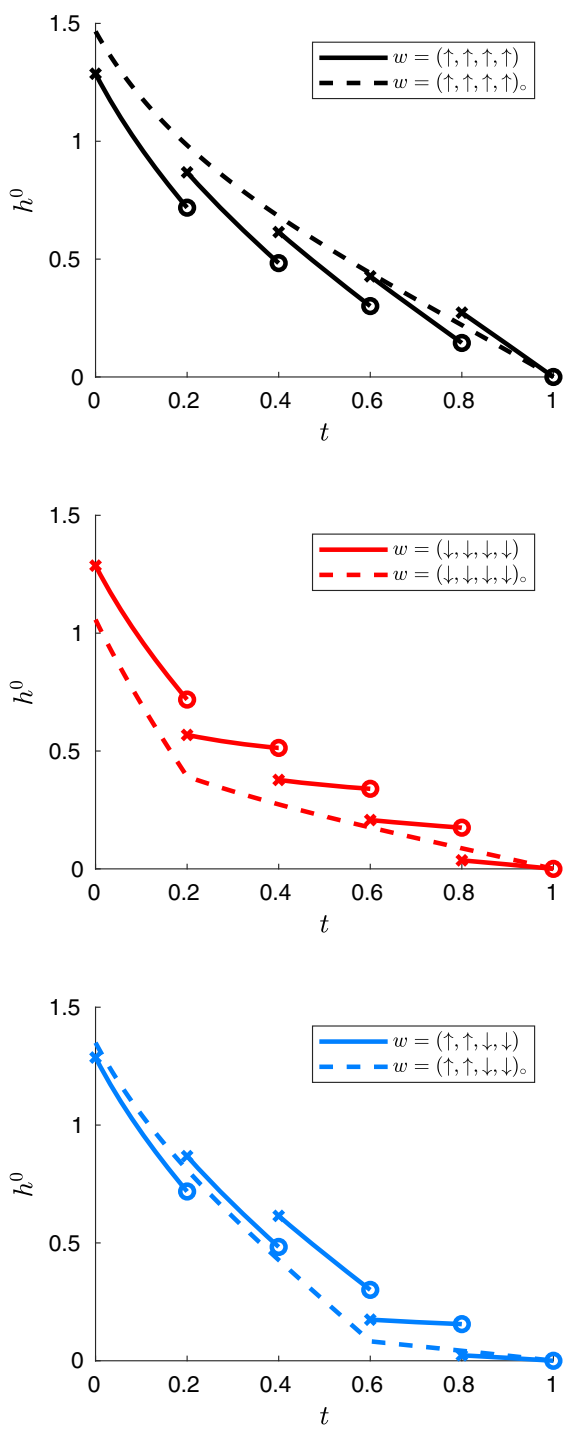

with catastrophe $(\mathrm{C})$
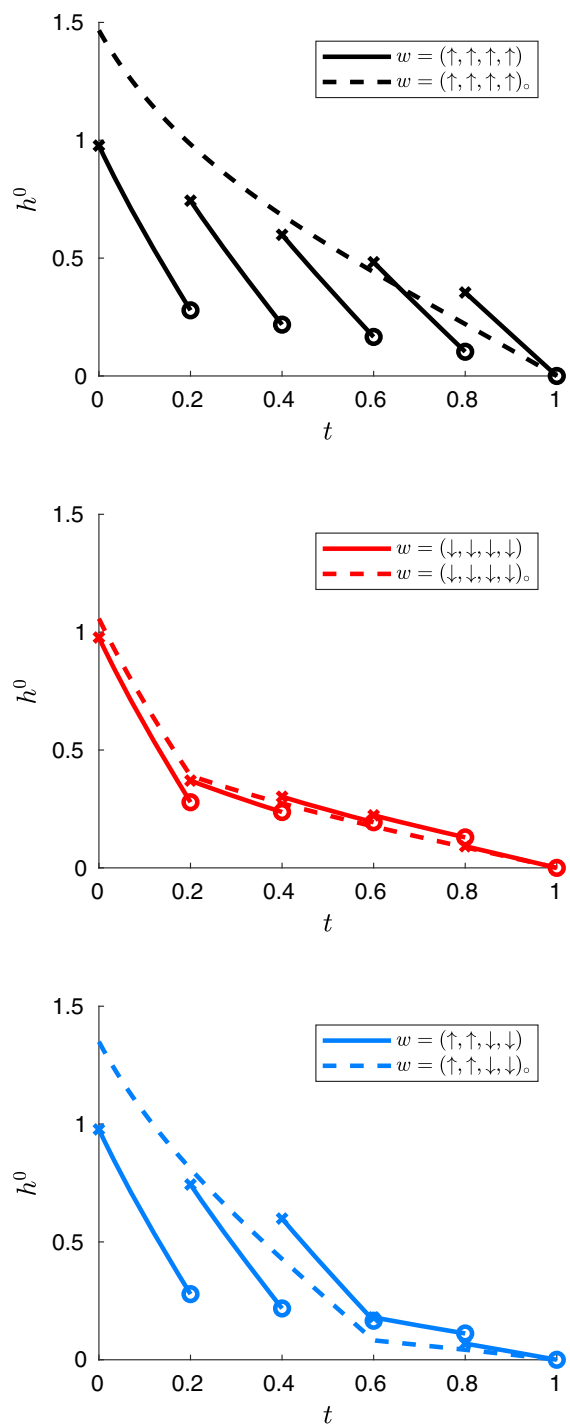

Fig. 4 Optimal action $h^{0}$ of non-farmers in models (nC) and (C)

The infection rate is proportional to the prevalence of the disease, i.e. the percentage of currently infected agents. Susceptible agents can make individual efforts of size $u \in \mathbb{U} \triangleq[0,1]$ to protect themselves against infection and thus reduce intensity of infection. The transition intensities are given by 
without catastrophe $(\mathrm{nC})$
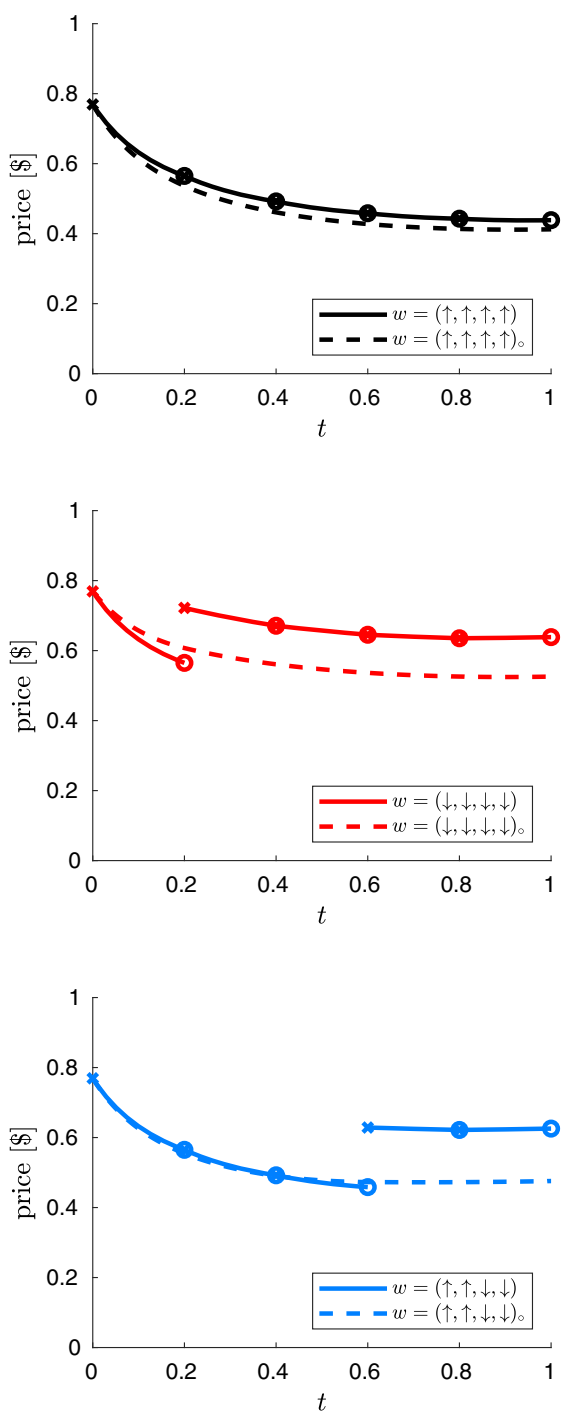

with catastrophe $(\mathrm{C})$
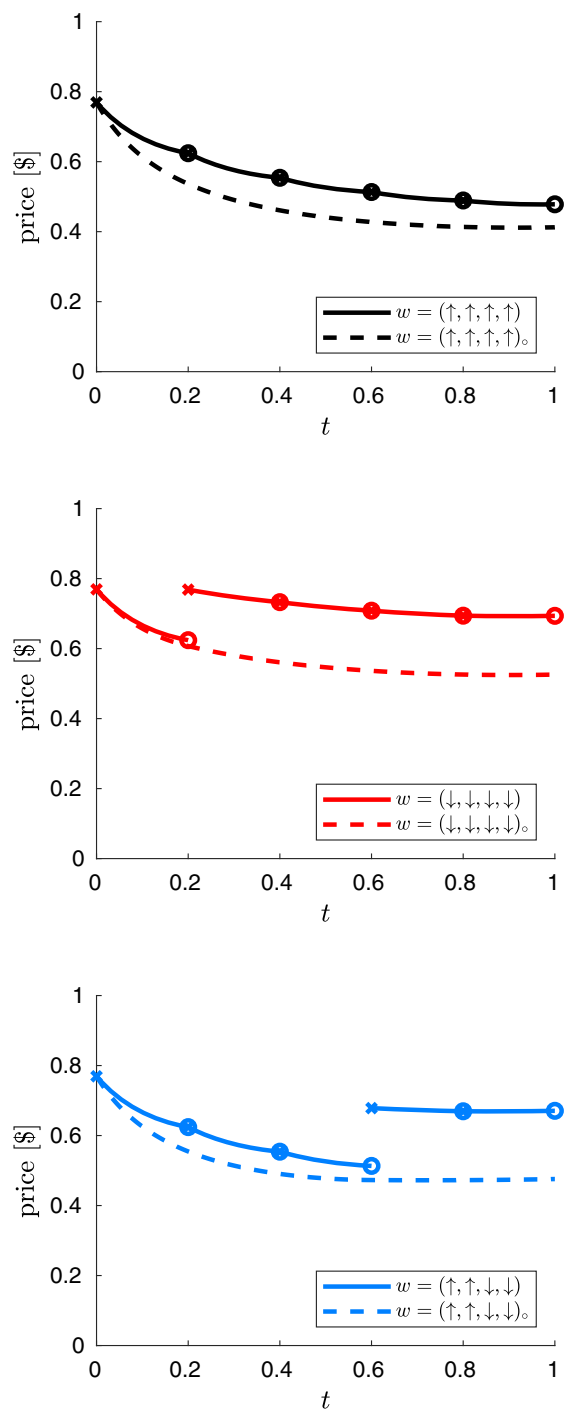

Fig. 5 Equilibrium market prices in models (nC) and (C)

$$
Q(t, w, m, u) \triangleq\left[\begin{array}{ccc}
-q_{\mathrm{inf}}(t, w, m, u) & q_{\mathrm{inf}}(t, w, m, u) & 0 \\
0 & -q_{\mathrm{IR}} & q_{\mathrm{IR}} \\
0 & 0 & 0
\end{array}\right]
$$

for $(t, w, m, u) \in[0, T] \times \mathbb{W}^{n} \times \mathbb{M} \times \mathbb{U}$, where $q_{\mathrm{IR}} \geq 0$ denotes the recovery rate of infected agents and the infection rate is given by 


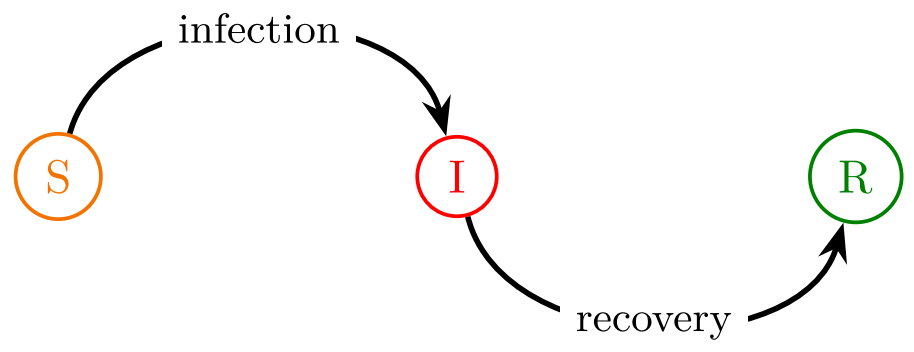

Fig. 6 State space and transitions in the SIR model

$$
q_{\text {inf }}(t, w, m, u) \triangleq q_{\mathrm{SI}} \cdot m^{\mathrm{I}} \cdot(1-u) \cdot \mathbb{1}_{\left\{t<\tau^{\star}\right\}}(w)
$$

with a given maximum rate $q_{\mathrm{SI}} \geq 0$. The running reward penalizes both protection efforts and time spent in the infected state; with $c_{\mathrm{P}}, \psi_{\mathrm{I}} \geq 0$ we set

$$
\psi^{\mathrm{S}}(t, w, m, u) \triangleq-c_{\mathrm{P}} \frac{u}{1-u}, \quad \psi^{\mathrm{I}}(t, w, m, u) \triangleq-\psi_{\mathrm{I}}, \quad \psi^{\mathrm{R}}(t, w, m, u) \triangleq 0 .
$$

In addition, we include the possibility of a one-shot vaccination that becomes available, simultaneously to all agents, at a random point of time $\tau^{\star} \in\left\{T_{1}, \ldots, T_{n}\right\} \subset$ $(0, T)$. We set $\mathbb{W} \triangleq\{0,1\}$ and identify the $k^{\text {th }}$ unit vector $e_{k}=\left(\delta_{k j}\right)_{j \in[1: n]} \in \mathbb{W}^{n}$, $k \in[1: n]$ with the indicator of the event $\left\{\tau^{\star}=T_{k}\right\}$. The event that no vaccine is available until $T$ is represented by $0 \in \mathbb{W}^{n}$; we set $\tau^{\star} \triangleq+\infty$ in this case. ${ }^{10}$ If and when it is available, all susceptible agents are vaccinated instantaneously, rendering them immune to infection; thus

$$
J^{\mathrm{S}}(t, w, m) \triangleq\left\{\begin{array}{ll}
\mathrm{R} & \text { if } t \in\left\{T_{1}, \ldots, T_{n}\right\}, t=T_{k}=\tau^{\star}, \\
\mathrm{S} & \text { otherwise }
\end{array} \text { and } J^{i}(t, w, m) \triangleq i \text { for } i \in\{\mathrm{I}, \mathrm{R}\} .\right.
$$

The probability of vaccination becoming available is proportional to the percentage of agents that have already recovered from the disease. Thus for $k \in[1: n], w_{1}, \ldots, w_{k} \in$ $\mathbb{W}$ and $m \in \mathbb{M}$ we set

$$
\kappa_{k}\left(1 \mid w_{1}, \ldots, w_{k-1}, m\right) \triangleq \begin{cases}\alpha \cdot m^{\mathrm{R}} & \text { if } w_{1}, \ldots, w_{k-1}=0 \\ 0 & \text { otherwise }\end{cases}
$$

and $\kappa_{k}\left(0 \mid w_{1}, \ldots, w_{k-1}, m\right) \triangleq 1-\kappa_{k}\left(1 \mid w_{1}, \ldots, w_{k-1}, m\right)$ where $\alpha \in(0,1]$. As a consequence, for all $(i, t, w, m, v) \in \mathbb{S} \times[0, T] \times \mathbb{W} \times \mathbb{M} \times \mathbb{R}^{3}$, a maximizer as

\footnotetext{
$\overline{10}$ The specification of $\kappa_{k}, k \in[1: n]$, below implies that $\tau^{\star}=+\infty$ is equivalent to $w=0 \in \mathbb{W}^{n} \mathbb{P}$-a.s., i.e., the configurations $\mathbb{W}^{n} \backslash\left(\{0\} \cup\left\{e_{k}: k \in[1: n]\right\}\right)$ are $\mathbb{P}$-negligible.
} 
Table 2 Coefficients in the SIR model

\begin{tabular}{lllllllll}
\hline Parameter & $T$ & $n$ & $T_{k}$ & $\alpha$ & $q_{\mathrm{SI}}$ & $q_{\mathrm{IR}}$ & $c_{\mathrm{P}}$ & $\psi_{\mathrm{I}}$ \\
\hline Value & 20 & 1999 & $k \cdot 0.01$ & 0.1 & 7.5 & 0.5 & 25 & 100 \\
\hline
\end{tabular}

required in Assumption 3 is given by ${ }^{11}$

$$
h^{\mathrm{S}}(t, w, m, v) \triangleq\left\{\begin{array}{cl}
{\left[1-\sqrt{\frac{c_{\mathrm{P}}}{q_{\mathrm{SI} \cdot m^{\mathrm{I}} \cdot\left(v^{\mathrm{S}}-v^{\mathrm{I}}\right)}}}\right]^{+}} & \text {if } v^{\mathrm{S}}>v^{\mathrm{I}}, m^{\mathrm{I}}>0 \text { and } t<\tau^{\star} \\
0 & \text { otherwise }
\end{array}\right.
$$

and $h^{i}(t, w, m, v) \triangleq 0$ for $i \in\{\mathrm{I}, \mathrm{R}\}$.

Remark 17 (SIR Models in the Literature). Note that, given the above specification of the transition matrix $Q$, the forward dynamics (E1) within the equilibrium system (E1)-(E6) read as follows:

$$
\left\{\begin{aligned}
& \dot{\mu}^{\mathrm{S}}(t, w)=-q_{\mathrm{SI}} \cdot \mu^{\mathrm{I}}(t, w) \cdot\left(1-h^{\mathrm{S}}(t, w, \mu(t, w), v(t, w))\right) \cdot \mathbb{1}_{\left\{t<\tau^{\star}\right\}}(w) \cdot \mu^{\mathrm{S}}(t, w) \\
& \dot{\mu}^{\mathrm{I}}(t, w)=q_{\mathrm{SI}} \cdot \mu^{\mathrm{I}}(t, w) \cdot\left(1-h^{\mathrm{S}}(t, w, \mu(t, w), v(t, w))\right) \cdot \mathbb{1}_{\left\{t<\tau^{\star}\right\}}(w) \cdot \mu^{\mathrm{S}}(t, w)-q_{\mathrm{IR}} \cdot \mu^{\mathrm{I}}(t, w) \\
& \dot{\mu}^{\mathrm{R}}(t, w)= q_{\mathrm{IR}} \cdot \mu^{\mathrm{I}}(t, w) .
\end{aligned}\right.
$$

Disregarding common noise, these constitute a ramification of the classical SIR dynamics, which are a basic building block of numerous compartmental epidemic models in the literature; see, among others, [32,37,38,47] and the references therein. The SIR mean field game with controlled infection rates, albeit without common noise, has recently been studied in the independent article [26]; we also refer to [46] and [23] for mean field models with controlled vaccination rates. Mathematically similar contagion mechanisms also appear in, e.g., [40,41], §7.2.3 in [9], §7.1.10 in [10], or §4.4 in [52].

While Theorem 13 guarantees existence of a mean field equilibrium for (a variant ${ }^{12}$ of) the SIR model, the monotonicity conditions of Theorem 16 do not hold in this setup. ${ }^{13}$ Nevertheless, our numerical results reliably yield consistent equilibria. For our illustrations, the initial distribution of agents is given by $m_{0} \triangleq(0.995,0.005,0.00)$, and the model coefficients are reported in Table 2. Note that there are $n=1999$ common noise times $T_{k}=k \cdot 0.01, k=1, \ldots, 1999$, at which a vaccine can be administered. The specifications of $q_{\mathrm{SI}}$ and $q_{\mathrm{IR}}$ imply a basic reproduction number $R_{0} \triangleq q_{\mathrm{SI}} / q_{\mathrm{IR}}=15$ in the absence of vaccination and protection efforts.

Our results for the mean field equilibrium distributions of agents $\mu$ and the corresponding optimal protection efforts of susceptible agents $h^{\mathrm{S}}$ are displayed in Figs. 7, 8 , and 9 for different common noises configurations, i.e. vaccination times $\tau^{\star}$. As in

\footnotetext{
11 Note that for given $w \in \mathbb{W}^{n}$ the stated maximizer $h^{\mathrm{S}}$ is unique for times $t<\tau^{\star}$; otherwise its specification is immaterial. The latter applies likewise to $h^{\mathrm{I}}$ and $h^{\mathrm{R}}$.

12 With action space $\mathbb{U} \triangleq\left[0, u_{\max }\right]$ for an arbitrary, but fixed $u_{\max }<1$.

13 In fact, Assumption 14(iii) is not even satisfied in the baseline SIR model without protection or vaccination.
} 
$w$ : no vaccination
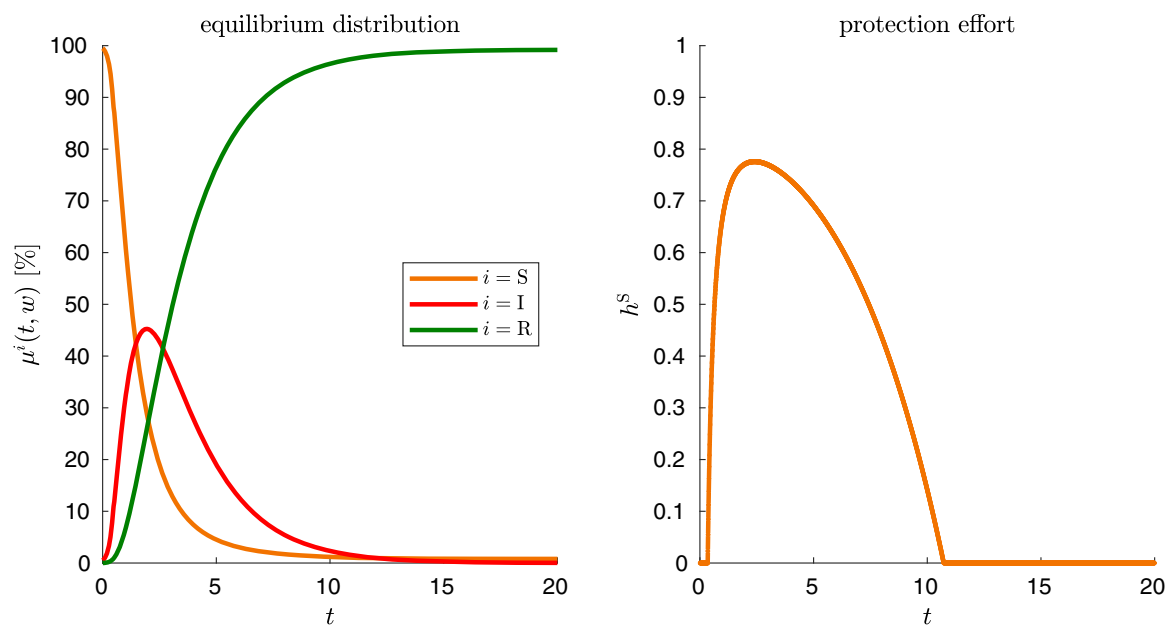

$w_{\circ}:$ no vaccination
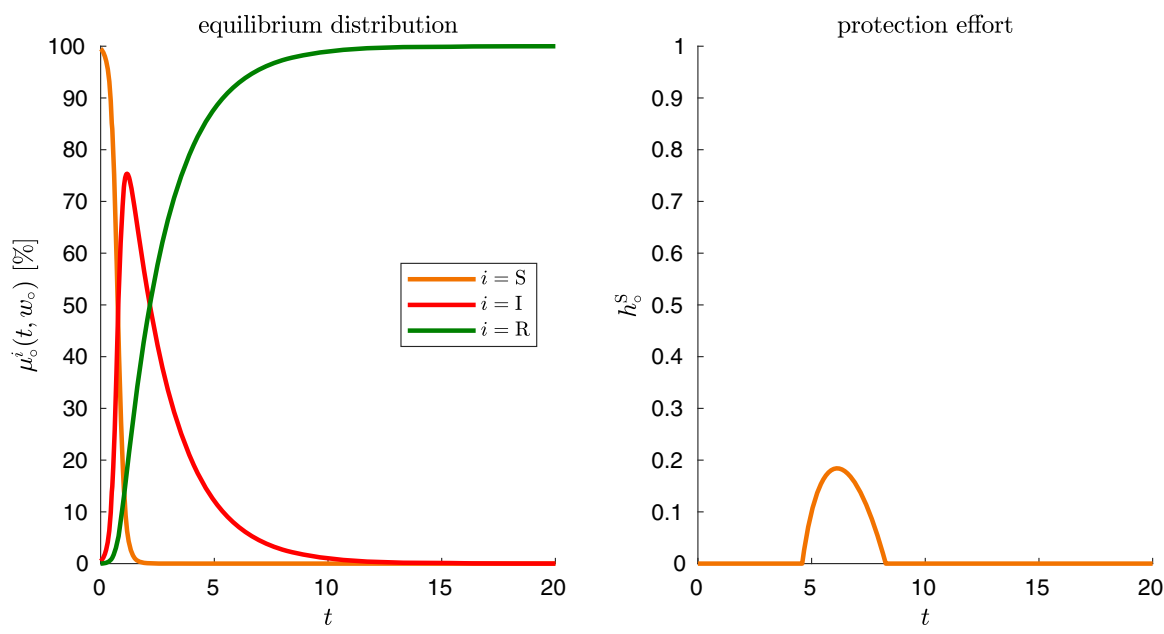

Fig. 7 Equilibrium distribution and protection effort for $\tau^{\star}=+\infty$ : Mean field game with common noise (top) and corresponding perfect-foresight equilibrium (bottom)

Sect. 5.1, we also display the corresponding (theoretical) perfect-foresight equilibria, marked by the subscript 0 .

Note that an agent's running reward is the same in state $\mathrm{S}$ with zero protection effort and in state R; agents are penalized relative to these in state I and hence aim to avoid that state. Susceptible agents can reach the state R of immunity by two ways: First, they can become infected and overcome the disease; second, they can be vaccinated and jump instantly from state $\mathrm{S}$ to state $\mathrm{R}$. While the first alternative is painful, the second comes at no cost and is hence clearly preferable. However, as the availability of 
$w:$ vaccination in $\tau^{\star}=2.5$
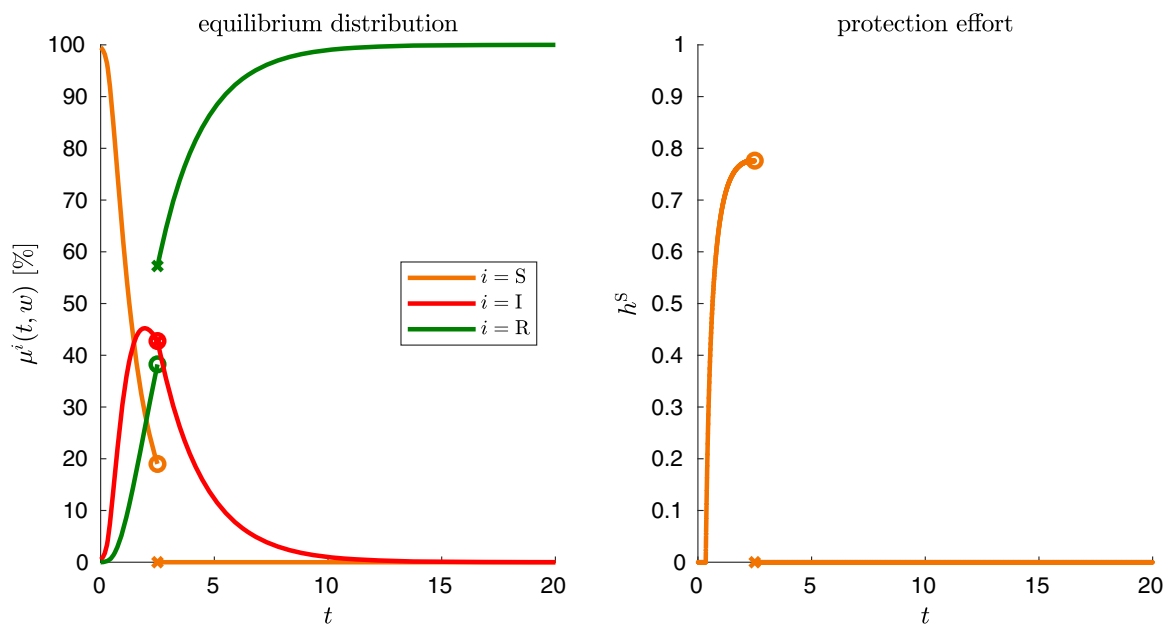

$w_{\circ}$ : vaccination in $\tau^{\star}=2.5$
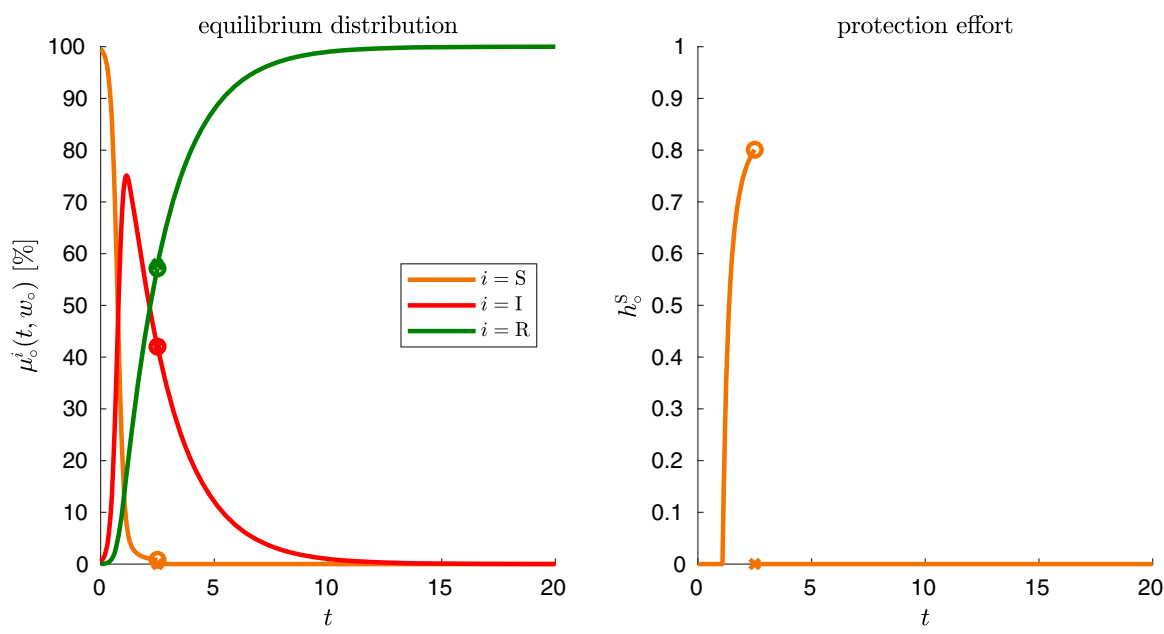

Fig. 8 Equilibrium distribution and protection effort for $\tau^{\star}=2.5$ : Mean field game with common noise (top) and corresponding perfect-foresight equilibrium (bottom)

a vaccine cannot be directly controlled by the agents, they can only protect themselves against infection at a certain running cost until the vaccine becomes available.

Figures 7, 8, and 9 demonstrate that the possibility of vaccination as a common noise event can dampen the spread of the disease and lower the peak infection rate. This is due to an increase in agents' protection efforts during the time period when the proportion of infected agents is high. By contrast, in the perfect-foresight equilibria where the vaccination date is known, agents do not make substantial protection efforts until the vaccination date is imminent, see Figs. 8 and 9; in the scenario without vaccination, see Fig. 7, protection efforts are only ever made by a very small fraction 
$w:$ vaccination in $\tau^{\star}=5$
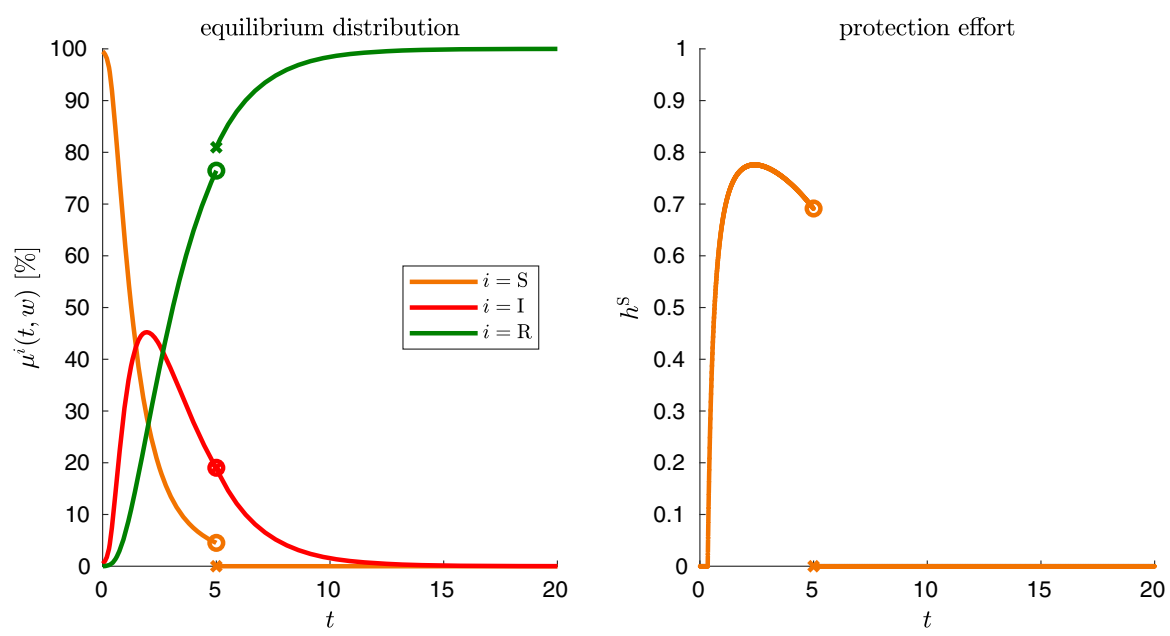

$w_{\circ}$ : vaccination in $\tau^{\star}=5$
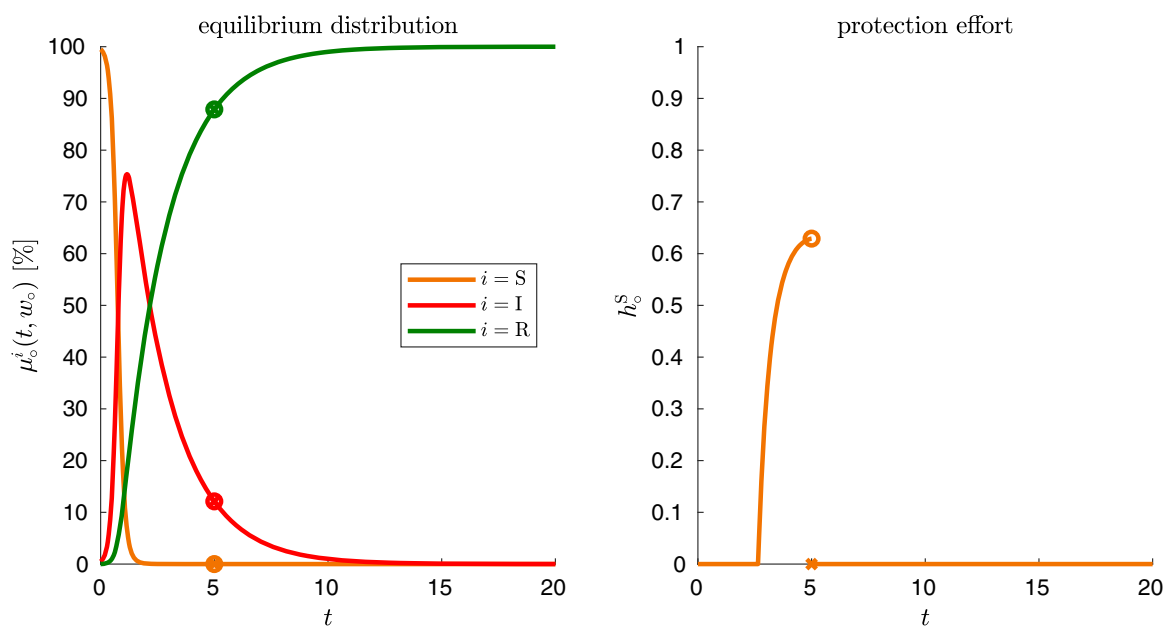

Fig. 9 Equilibrium distribution and protection effort for $\tau^{\star}=5$ : Mean field game with common noise (top) and corresponding perfect-foresight equilibrium (bottom)

of the population. With perfect foresight, the agents' main rationale is to avoid being in state I when the vaccine becomes available. This highlights the importance of being able to model the vaccination date as a (random) common noise event. Finally, observe that our numerical results indicate convergence to the stationary distribution $\bar{\mu}=(0,0,1) \in \mathbb{M}$, showing that the model is able to capture the entire evolution of an epidemic.

Acknowledgements We thank the anonymous referees for valuable comments and suggestions. Daniel Hoffmann and Frank Seifried gratefully acknowledge financial support from the German Research Foundation (DFG) within the Research Training Group 2126: Algorithmic Optimization. 
Funding Open Access funding enabled and organized by Projekt DEAL.

Open Access This article is licensed under a Creative Commons Attribution 4.0 International License, which permits use, sharing, adaptation, distribution and reproduction in any medium or format, as long as you give appropriate credit to the original author(s) and the source, provide a link to the Creative Commons licence, and indicate if changes were made. The images or other third party material in this article are included in the article's Creative Commons licence, unless indicated otherwise in a credit line to the material. If material is not included in the article's Creative Commons licence and your intended use is not permitted by statutory regulation or exceeds the permitted use, you will need to obtain permission directly from the copyright holder. To view a copy of this licence, visit http://creativecommons.org/licenses/by/4.0/.

\section{A Appendix: Proof of Theorem 13}

Let $E \subseteq \mathbb{R}^{d}$ and define the space

$$
\mathrm{D}(E) \triangleq\left\{f:[0, T] \times \mathbb{W}^{n} \rightarrow E: f \text { is càdlàg and non-anticipative }\right\}
$$

together with the norm $\|f\|_{\text {sup }} \triangleq \sup _{t \in[0, T], w \in \mathbb{W}^{n}}\|f(t, w)\|$ for $f \in \mathrm{D}(E)$. It is clear that $\mathrm{D}(E)$ is a Banach space provided $E \subseteq \mathbb{R}^{d}$ is closed; the linear subspace of regular non-anticipative functions is denoted by

$$
\operatorname{Reg}(E) \triangleq\{f \in \mathrm{D}(E): f \text { is regular }\} .
$$

Lemma A.1 (Backward Gronwall estimate) Let $f \in D([0, \infty))$ and $\alpha, \beta, \vartheta, \rho, \eta \geq 0$. Suppose that $f(T, w) \leq \rho \cdot \eta$ for all $w \in \mathbb{W}^{n}$,

$$
\begin{aligned}
& f(t, w) \leq f\left(T_{k+1}-, w\right)+\alpha\left(T_{k+1}-t\right) \cdot \eta+\beta \cdot \int_{t}^{T_{k+1}} f(s, w) \mathrm{d} s, \\
& t \in\left[T_{k}, T_{k+1}\right\rangle, w \in \mathbb{W}^{n},
\end{aligned}
$$

for $k \in[0: n]$, and

$$
f\left(T_{k}-, w\right) \leq \sum_{\bar{w}_{k} \in \mathbb{W}} \gamma_{k}\left(w_{-k}, \bar{w}_{k}\right) \cdot f\left(T_{k},\left(w_{-k}, \bar{w}_{k}\right)\right)+\vartheta \cdot \eta, \quad w \in \mathbb{W}^{n},
$$

for $k \in[1: n]$, where for all $w_{1}, \ldots, w_{k-1} \in \mathbb{W}$ the family $\left\{\gamma_{k}\left(w_{-k}, \bar{w}_{k}\right)\right\}_{\bar{w}_{k} \in \mathbb{W}}$ consists of probability weights on $\mathbb{W}$. Then we have

$$
\begin{gathered}
f(t, w) \leq C \cdot \eta \text { for all }(t, w) \in[0, T] \times \mathbb{W}^{n}, \\
\text { where } \quad C \triangleq(\rho+\alpha T+(n+1) \vartheta) \cdot \mathrm{e}^{\beta T} .
\end{gathered}
$$

Proof We recursively define $C_{n+1} \triangleq 1$ and

$$
C_{k} \triangleq\left(C_{k+1}+\alpha\left(T_{k+1}-T_{k}\right)+\vartheta\right) \cdot \mathrm{e}^{\beta\left(T_{k+1}-T_{k}\right)} \quad \text { for } k \in[0: n]
$$


and note that $C_{n+1} \leq C_{n} \leq \cdots \leq C_{1} \leq C_{0} \leq C$. Hence it suffices to show that

$$
f(t, w) \leq C_{k} \cdot \eta \text { for all }(t, w) \in\left[T_{k}, T_{k+1}\right\rangle \times \mathbb{W}^{n}, k \in[0: n]
$$

By assumption, $f\left(T_{n+1}-, w\right)=f(T, w) \leq \rho \cdot \eta$ for all $w \in \mathbb{W}^{n}$. Next let $k \in[0: n]$ and assume that

$$
f\left(T_{k+1}-, w\right) \leq C_{k+1} \cdot \eta \text { for all } w \in \mathbb{W}^{n}
$$

It follows from (28) and Gronwall's inequality on $\left[T_{k}, T_{k+1}\right\rangle$ that for all $w \in \mathbb{W}^{n}$

$$
\begin{aligned}
f(t, w) & \leq\left(f\left(T_{k+1}-, w\right)+\alpha\left(T_{k+1}-t\right) \cdot \eta\right) \cdot \mathrm{e}^{\beta\left(T_{k+1}-t\right)} \\
& \leq\left(C_{k+1}+\alpha\left(T_{k+1}-T_{k}\right)\right) \cdot \mathrm{e}^{\beta\left(T_{k+1}-T_{k}\right)} \cdot \eta .
\end{aligned}
$$

In particular, we have $f(t, w) \leq C_{k} \cdot \eta$ for $t \in\left[T_{k}, T_{k+1}\right\rangle$, and by (29)

$$
\begin{aligned}
f\left(T_{k}-, w\right) & \leq \sum_{\bar{w}_{k} \in \mathbb{W}} \gamma_{k}\left(w_{1}, \ldots, w_{k-1}, \bar{w}_{k}\right) \cdot f\left(T_{k},\left(w_{-k}, \bar{w}_{k}\right)\right)+\vartheta \cdot \eta \\
& \leq\left(C_{k+1}+\alpha\left(T_{k+1}-T_{k}\right)+\vartheta\right) \cdot \mathrm{e}^{\beta\left(T_{k+1}-T_{k}\right)} \cdot \eta=C_{k} \cdot \eta .
\end{aligned}
$$

Hence (30) follows by backward induction on $k=n, n-1, \ldots, 0$.

In the following, we first consider the backward system (E2), (E4), (E6) and subsequently the forward system (E1), (E3), (E5).

Lemma A.2 Suppose that Assumption 11 holds and let $\mu \in D(\mathbb{M})$. Then there exists a unique solution $\bar{v}$ of (E2) subject to (E4) and (E6). Moreover, we have $\bar{v} \in \operatorname{Reg}\left(\mathbb{R}^{d}\right)$ and $\|\bar{v}(t, w)\| \leq v_{\max }$ for all $(t, w) \in[0, T] \times \mathbb{W}^{n}$.

Proof Step 1: Construction of $\bar{v}$. We construct $\bar{v}$ by backward induction on $k \in$ $[0: n]$ on each segment $\left[T_{k}, T_{k+1}\right\rangle \times \mathbb{W}^{n}$. First, we set $\bar{v}(T, w) \triangleq \Psi(w, \mu(T, w))$ for $w \in \mathbb{W}^{n}$. Suppose that $k \in[0: n]$, fix $w \in \mathbb{W}^{n}$, and let $\tilde{v}\left(T_{k+1}, w_{T_{k}}\right) \in \mathbb{R}^{d}$ be given and independent of $w_{k+1}, \ldots, w_{n}$. Using Assumptions 11(i)-(ii) it follows that the Carathéodory conditions are satisfied, so [36, Theorem I.5.3] yields the unique Carathéodory solution $\tilde{v}\left(\cdot, w_{T_{k}}\right):\left[T_{k}, T_{k+1}\right] \rightarrow \mathbb{R}^{d}$ of

$$
\begin{aligned}
\tilde{v}\left(t, w_{T_{k}}\right)=\tilde{v}\left(T_{k+1}, w_{T_{k}}\right)+ & \int_{t}^{T_{k+1}}\left(\widehat{\psi}\left(s, w_{T_{k}}, \mu\left(s, w_{T_{k}}\right), \tilde{v}\left(s, w_{T_{k}}\right)\right)\right. \\
& \left.+\widehat{Q}\left(s, w_{T_{k}}, \mu\left(s, w_{T_{k}}\right), \tilde{v}\left(s, w_{T_{k}}\right)\right) \cdot \tilde{v}\left(s, w_{T_{k}}\right)\right) \mathrm{d} s \\
=\tilde{v}\left(T_{k+1}, w_{T_{k}}\right)+ & \int_{t}^{T_{k+1}}\left(\widehat{\psi}\left(s, w, \mu(s, w), \tilde{v}\left(s, w_{T_{k}}\right)\right)\right. \\
& \left.+\widehat{Q}\left(s, w, \mu(s, w), \tilde{v}\left(s, w_{T_{k}}\right)\right) \cdot \tilde{v}\left(s, w_{T_{k}}\right)\right) \mathrm{d} s, \quad t \in\left[T_{k}, T_{k+1}\right],
\end{aligned}
$$


where the final identity is due to the fact that $\widehat{\psi}(\cdot, \cdot, \bar{m}, \bar{v})$ and $\widehat{Q}(\cdot, \cdot, \bar{m}, \bar{v})$ are non-anticipative. Define

$$
\bar{v}(t, w) \triangleq \tilde{v}\left(t, w_{T_{k}}\right) \quad \text { for } t \in\left[T_{k}, T_{k+1}\right\rangle \quad \text { and each } w \in \mathbb{W}^{n} .
$$

By construction, $\bar{v}(\cdot, w)$ solves $(\mathrm{E} 2)$ on $\left[T_{k}, T_{k+1}\right\rangle$ and does not depend on $w_{k+1}, \ldots, w_{n}$. Having constructed $\bar{v}$ on $\left[T_{k}, T_{k+1}\right\rangle \times \mathbb{W}^{n}$, we use (E4) and define

$$
\bar{v}\left(T_{k}, w_{T_{k-1}}\right) \triangleq \Psi_{k}\left(w, \mu\left(T_{k}-, w\right), \bar{v}\left(T_{k}, \cdot\right)\right)
$$

for $w \in \mathbb{W}^{n}$. By (10) and the fact that $\mu$ and $J$ are non-anticipative, it follows that this definition does not depend on $w_{k}, \ldots, w_{n}$. Consequently, the above construction can be iterated, and hence we obtain $\bar{v}$ as the unique solution of (E2) subject to (E4) and (E6). By definition, $\bar{v}$ is non-anticipative and regular, i.e. $\bar{v} \in \operatorname{Reg}\left(\mathbb{R}^{d}\right)$.

Step 2: A priori bound. For $k \in[0: n]$ and $(t, w) \in\left[T_{k}, T_{k+1}\right\rangle \times \mathbb{W}^{n}$ we have

$$
\begin{aligned}
\|\bar{v}(t, w)\| \leq & \left\|\bar{v}\left(T_{k+1}-, w\right)\right\|+\int_{t}^{T_{k+1}}\|\widehat{\psi}(s, w, \mu(s, w), \bar{v}(s, w))\| \\
& +\|\widehat{Q}(s, w, \mu(s, w), \bar{v}(s, w))\| \cdot\|\bar{v}(s, w)\| \mathrm{d} s \\
& \leq\left\|\bar{v}\left(T_{k+1}-, w\right)\right\|+\psi_{\max } \cdot\left(T_{k+1}-t\right)+Q_{\max } \cdot \int_{t}^{T_{k+1}}\|\bar{v}(s, w)\| \mathrm{d} s .
\end{aligned}
$$

On the other hand, for $k \in[1: n], w \in \mathbb{W}^{n}$ and $i \in \mathbb{S}$ we observe from (10) that

$$
\left\|\bar{v}\left(T_{k}-, w\right)\right\| \leq \sum_{\bar{w}_{k} \in \mathbb{W}} \kappa_{k}\left(\bar{w}_{k} \mid w_{1}, \ldots, w_{k-1}, \mu\left(T_{k}-, w_{T_{k}-}\right)\right) \cdot \| \bar{v}\left(T_{k},\left(w_{-k}, \bar{w}_{k}\right) \| .\right.
$$

Since $\|\bar{v}(T, w)\|=\|\Psi(w, \mu(T, w))\| \leq \Psi_{\max }$ it follows from (31), (32) and Lemma A.1 with $\eta \triangleq \psi_{\max }, \vartheta \triangleq 0$ and $\rho \triangleq \Psi_{\max } / \psi_{\max }$ that

$$
\|\bar{v}(t, w)\| \leq C \cdot \eta \leq\left(\Psi_{\max }+T \cdot \psi_{\max }\right) \cdot \mathrm{e}^{Q_{\max } \cdot T}=v_{\max } \quad \text { for all }(t, w) \in[0, T] \times \mathbb{W}^{n}
$$

Lemma A.3 Suppose that Assumption 11 is satisfied and let $v \in D\left(\mathbb{R}^{d}\right)$. Then there is a unique solution $\bar{\mu}$ of (E1) subject to (E3) and (E5), and we have $\bar{\mu} \in \operatorname{Reg}(\mathbb{M})$.

Proof The proof is analogous to (but somewhat simpler than) that of Lemma A.2.

Proof of Theorem 13 We divide the proof into four steps:

Step 1: Solution operators. We define

$$
\overleftarrow{\chi}: \mathrm{D}(\mathbb{M}) \rightarrow \operatorname{Reg}\left(\mathbb{R}^{d}\right), \quad \overleftarrow{\chi}[\mu] \triangleq \bar{v}
$$


where $\bar{v} \in \operatorname{Reg}\left(\mathbb{R}^{d}\right)$ is the unique solution of (E2) subject to (E4) and (E6) given $\mu \in \mathrm{D}(\mathbb{M}) ; \overleftarrow{\chi}$ is well-defined by Lemma A.2. Moreover, let

$$
\vec{\chi}: \mathrm{D}\left(\mathbb{R}^{d}\right) \rightarrow \operatorname{Reg}(\mathbb{M}), \quad \vec{\chi}[v] \triangleq \bar{\mu}
$$

where $\bar{\mu} \in \operatorname{Reg}(\mathbb{M})$ is the unique solution of (E1) subject to (E3) and (E5) given $v \in \mathrm{D}\left(\mathbb{R}^{d}\right) ; \vec{\chi}$ is well-defined by Lemma A.3.

Step 2: Continuity of $\overleftarrow{\chi}$. Let $\mu_{0} \in \mathrm{D}(\mathbb{M})$, set $\bar{v}_{0} \triangleq \overleftarrow{\chi}\left[\mu_{0}\right]$ and fix some $\varepsilon>0$. We set

$$
\begin{aligned}
& \alpha \triangleq L_{\widehat{\psi}}+L_{\widehat{Q}} \cdot v_{\max }, \quad \beta \triangleq L_{\widehat{\psi}}+Q_{\max }+L_{\widehat{Q}} \cdot v_{\max }, \\
& C \triangleq(\alpha T+n+2) \cdot \mathrm{e}^{\beta T} \quad \text { and } \quad \eta \triangleq \frac{\varepsilon}{C} .
\end{aligned}
$$

By Assumptions 11(iii)-(iv), for each $k \in[1: n]$ we can pick $\delta_{k}>0$ such that

$$
\begin{aligned}
& \mid \sum_{\bar{w}_{k} \in \mathbb{W}}\left(\kappa_{k}\left(\bar{w}_{k} \mid w_{1}, \ldots, w_{k-1}, m\right) \bar{v}_{0}^{J^{i}\left(T_{k},\left(w_{-k}, \bar{w}_{k}\right), m\right)}\left(T_{k},\left(w_{-k}, \bar{w}_{k}\right)\right)\right. \\
& \left.\quad-\kappa_{k}\left(\bar{w}_{k} \mid w_{1}, \ldots, w_{k-1}, \mu_{0}\left(T_{k}-, w\right)\right) \bar{v}_{0}^{J^{i}\left(T_{k},\left(w_{-k}, \bar{w}_{k}\right), \mu_{0}\left(T_{k}-, w\right)\right)}\left(T_{k},\left(w_{-k}, \bar{w}_{k}\right)\right)\right) \mid \leq \eta
\end{aligned}
$$

for all $i \in \mathbb{S}$ and $(m, w) \in \mathbb{M} \times \mathbb{W}^{n}$ with $\left\|m-\mu_{0}\left(T_{k}-, w\right)\right\| \leq \delta_{k}$,

and $\delta_{n+1}>0$ such that

$$
\begin{aligned}
& \left\|\Psi(w, m)-\Psi\left(w, \mu_{0}(T, w)\right)\right\| \leq \eta \\
& \quad \text { for all }(m, w) \in \mathbb{M} \times \mathbb{W}^{n} \text { with }\left\|m-\mu_{0}(T, w)\right\| \leq \delta_{n+1} .
\end{aligned}
$$

We define $\delta>0$ via

$$
\delta \triangleq \eta \wedge \delta_{1} \wedge \cdots \wedge \delta_{n+1}
$$

and let $\mu \in \mathrm{D}(\mathbb{M})$ such that $\left\|\mu-\mu_{0}\right\|_{\text {sup }} \leq \delta$; set $\bar{v} \triangleq \overleftarrow{\chi}[\mu]$. For each $w \in \mathbb{W}^{n}$, it follows from Assumptions 11(i)-(ii) and (35) that for all $t \in\left[T_{k}, T_{k+1}\right\rangle, k \in[0: n]$, we have

$$
\begin{aligned}
& \left\|\bar{v}(t, w)-\bar{v}_{0}(t, w)\right\| \leq\left\|\bar{v}\left(T_{k+1}-, w\right)-\bar{v}_{0}\left(T_{k+1}-, w\right)\right\| \\
& \quad+\int_{t}^{T_{k+1}}\left\|\widehat{\psi}(s, w, \mu(s, w), \bar{v}(s, w))-\widehat{\psi}\left(s, w, \mu_{0}(s, w), \bar{v}_{0}(s, w)\right)\right\| \mathrm{d} s \\
& \quad+\int_{t}^{T_{k+1}}\left\|\widehat{Q}(s, w, \mu(s, w), \bar{v}(s, w)) \cdot \bar{v}(s, w)-\widehat{Q}\left(s, w, \mu_{0}(s, w), \bar{v}_{0}(s, w)\right) \cdot \bar{v}_{0}(s, w)\right\| \mathrm{d} s \\
& \leq\left\|\bar{v}\left(T_{k+1}-, w\right)-\bar{v}_{0}\left(T_{k+1}-, w\right)\right\|+\left(L_{\widehat{\psi}}+L_{\widehat{Q}} \cdot v_{\max }\right) \cdot\left(T_{k+1}-t\right) \cdot \eta \\
& \quad+\left(L_{\widehat{\psi}}+Q_{\max }+L_{\widehat{Q}} \cdot v_{\max }\right) \cdot \int_{t}^{T_{k+1}}\left\|\bar{v}(s, w)-\bar{v}_{0}(s, w)\right\| \mathrm{d} s
\end{aligned}
$$




$$
=\left\|\bar{v}\left(T_{k+1}-, w\right)-\bar{v}_{0}\left(T_{k+1}-, w\right)\right\|+\alpha\left(T_{k+1}-t\right) \cdot \eta+\beta \cdot \int_{t}^{T_{k+1}}\left\|\bar{v}(s, w)-\bar{v}_{0}(s, w)\right\| \mathrm{d} s .
$$

Moreover, for $k \in[1: n]$ we obtain from (33) that

$$
\begin{aligned}
& \left\|\bar{v}\left(T_{k}-, w\right)-\bar{v}_{0}\left(T_{k}-, w\right)\right\| \\
& \leq \sum_{\bar{w}_{k} \in \mathbb{W}} \kappa_{k}\left(\bar{w}_{k} \mid w_{1}, \ldots, w_{k-1}, \mu\left(T_{k}-, w_{T_{k}-}\right)\right) \\
& \quad \cdot\left\|\bar{v}\left(T_{k},\left(w_{-k}, \bar{w}_{k}\right)\right)-\bar{v}_{0}\left(T_{k},\left(w_{-k}, \bar{w}_{k}\right)\right)\right\|+\eta
\end{aligned}
$$

and from (34) that

$$
\left\|\bar{v}(T, w)-\bar{v}_{0}(T, w)\right\|=\left\|\Psi(w, \mu(T, w))-\Psi\left(w, \mu_{0}(T, w)\right)\right\| \leq \eta
$$

In view of (36), (37) and (38), it follows from Lemma A.1 that

$$
\left\|\bar{v}(t, w)-\bar{v}_{0}(t, w)\right\| \leq C \cdot \eta=\varepsilon \quad \text { for all }(t, w) \in[0, T] \times \mathbb{W}^{n}
$$

i.e. $\left\|\overleftarrow{\chi}[\mu]-\overleftarrow{\chi}\left[\mu_{0}\right]\right\|_{\text {sup }}=\left\|\bar{v}-\bar{v}_{0}\right\|_{\text {sup }} \leq \varepsilon$. Thus $\overleftarrow{\chi}$ is continuous with respect to $\|\cdot\|_{\text {sup. }}$

Step 3: Continuity of $\vec{\chi}$. Let $v_{0} \in \mathrm{D}\left(\mathbb{R}^{d}\right)$, set $\bar{\mu}_{0} \triangleq \vec{\chi}\left[v_{0}\right]$ and fix some $\varepsilon>0$. We set $\delta_{n+1} \triangleq \varepsilon$ and $c \triangleq Q_{\max }+L_{\widehat{Q}}$ and recursively determine $\delta_{1}, \ldots, \delta_{n} \in(0, \varepsilon)$ using Assumption 11(v) such that

$$
\begin{aligned}
& \left\|\Phi_{k}(w, m)-\Phi_{k}\left(w, \bar{\mu}_{0}\left(T_{k}-, w\right)\right)\right\| \leq \frac{\delta_{k+1}}{2} \cdot \mathrm{e}^{-c\left(T_{k+1}-T_{k}\right)} \\
& \quad \text { for all }(m, w) \in \mathbb{M} \times \mathbb{W}^{n} \text { with }\left\|m-\bar{\mu}_{0}\left(T_{k}-, w\right)\right\| \leq \delta_{k} .
\end{aligned}
$$

We define $\delta>0$ by

$$
\delta \triangleq \frac{\mathrm{e}^{-c T}}{2 c T} \cdot \delta_{1} \wedge \cdots \wedge \delta_{n}
$$

and let $v \in \mathrm{D}\left(\mathbb{R}^{d}\right)$ such that $\left\|v-v_{0}\right\|_{\text {sup }} \leq \delta$; put $\bar{\mu} \triangleq \vec{\chi}[v]$. We fix $k \in[0: n]$ and suppose that

$$
\left\|\bar{\mu}\left(T_{k}, w\right)-\bar{\mu}_{0}\left(T_{k}, w\right)\right\| \leq \frac{\delta_{k+1}}{2} \cdot \mathrm{e}^{-c\left(T_{k+1}-T_{k}\right)}, \quad w \in \mathbb{W}^{n}
$$

For each $w \in \mathbb{W}^{n}$, we have from Assumption 11(ii)

$$
\begin{aligned}
& \left\|\bar{\mu}(t, w)-\bar{\mu}_{0}(t, w)\right\| \leq\left\|\bar{\mu}\left(T_{k}, w\right)-\bar{\mu}_{0}\left(T_{k}, w\right)\right\| \\
& \quad+\int_{T_{k}}^{t}\left\|\bar{\mu}(s, w) \cdot \widehat{Q}(s, w, \bar{\mu}(s, w), v(s, w))-\bar{\mu}_{0}(s, w) \cdot \widehat{Q}\left(s, w, \bar{\mu}_{0}(s, w), v_{0}(s, w)\right)\right\| \mathrm{d} s \\
& \leq\left\|\bar{\mu}\left(T_{k}, w\right)-\bar{\mu}_{0}\left(T_{k}, w\right)\right\|+c\left(t-T_{k}\right) \cdot\left\|v-v_{0}\right\|_{\text {sup }}+c \cdot \int_{T_{k}}^{t}\left\|\bar{\mu}(s, w)-\bar{\mu}_{0}(s, w)\right\| \mathrm{d} s
\end{aligned}
$$


on $\left[T_{k}, T_{k+1}\right\rangle$, so using Gronwall's inequality it follows that

$$
\left\|\bar{\mu}(t, w)-\bar{\mu}_{0}(t, w)\right\| \leq\left(\left\|\bar{\mu}\left(T_{k}, w\right)-\bar{\mu}_{0}\left(T_{k}, w\right)\right\|+c\left(t-T_{k}\right) \cdot\left\|v-v_{0}\right\|_{\text {sup }}\right) \cdot \mathrm{e}^{c\left(t-T_{k}\right)} .
$$

Since by (40) we have $c\left(t-T_{k}\right) \cdot \delta \cdot \mathrm{e}^{c\left(t-T_{k}\right)} \leq \frac{\delta_{k+1}}{2}$, we obtain

$$
\left\|\bar{\mu}(t, w)-\bar{\mu}_{0}(t, w)\right\| \leq \delta_{k+1} \quad \text { for all }(t, w) \in\left[T_{k}, T_{k+1}\right\rangle \times \mathbb{W}^{n}
$$

In particular, using (39) we conclude that

$$
\begin{aligned}
\left\|\bar{\mu}\left(T_{k+1}, w\right)-\bar{\mu}_{0}\left(T_{k+1}, w\right)\right\| & =\left\|\Phi_{k+1}\left(w, \bar{\mu}\left(T_{k+1}-, w\right)\right)-\Phi_{k+1}\left(w, \bar{\mu}_{0}\left(T_{k+1}-, w\right)\right)\right\| \\
& \leq \frac{\delta_{k+2}}{2} \cdot \mathrm{e}^{-c\left(T_{k+2}-T_{k+1}\right)}, \quad w \in \mathbb{W}^{n} .
\end{aligned}
$$

Since $\bar{\mu}(0, w)=\bar{\mu}_{0}(0, w)=m_{0}$ for all $w \in \mathbb{W}^{n}$, it follows by induction that (41) holds for all $k \in[0: n]$, and thus (42) implies

$$
\left\|\bar{\mu}(t, w)-\bar{\mu}_{0}(t, w)\right\| \leq \varepsilon \text { for all }(t, w) \in[0, T] \times \mathbb{W}^{n}
$$

Hence $\left\|\vec{\chi}[v]-\vec{\chi}\left[v_{0}\right]\right\|_{\text {sup }}=\left\|\bar{\mu}-\bar{\mu}_{0}\right\|_{\text {sup }} \leq \varepsilon$, so $\vec{\chi}$ is continuous with respect to $\|\cdot\|_{\text {sup. }}$

Step 4: Construction of the fixed point. Let $\chi: D(\mathbb{M}) \rightarrow \operatorname{Reg}(\mathbb{M}), \chi \triangleq \vec{\chi} \circ \overleftarrow{\chi}$ and note that $\chi$ is continuous with respect to $\|\cdot\|_{\text {sup }}$ by Steps 2 and 3 . We define

$$
\begin{aligned}
\operatorname{Lip}(\mathbb{M}) \triangleq\{\mu \in \mathrm{D}(\mathbb{M}): & \mu(\cdot, w) \text { is } Q_{\max }-\operatorname{Lipschitz} \\
& \text { on } \left.\left[T_{k}, T_{k+1}\right\rangle, k \in[0: n], \text { for all } w \in \mathbb{W}^{n}\right\}
\end{aligned}
$$

and note from $(\mathrm{E} 1)$ that $\chi: D(\mathbb{M}) \rightarrow \operatorname{Lip}(\mathbb{M})$, i.e. $\chi[\mu] \in \operatorname{Lip}(\mathbb{M})$ for every $\mu \in$ $\mathrm{D}(\mathbb{M})$. It is clear that $\operatorname{Lip}(\mathbb{M})$ is a non-empty, convex subset of $D(\mathbb{M})$; we now argue that $\operatorname{Lip}(\mathbb{M})$ is compact. Given a sequence $\left\{\mu_{\ell}\right\}_{\ell \in \mathbb{N}} \subseteq \operatorname{Lip}(\mathbb{M})$, for each $k \in[0: n]$ and $w \in \mathbb{W}^{n}$ we define

$$
\mu_{\ell}^{(k, w)}:\left[T_{k}, T_{k+1}\right] \rightarrow \mathbb{M}, \quad \mu_{\ell}^{(k, w)}(t) \triangleq \begin{cases}\mu_{\ell}(t, w) & \text { if } t \in\left[T_{k}, T_{k+1}\right\rangle \\ \mu_{\ell}\left(T_{k+1}-, w\right) & \text { if } t=T_{k+1}\end{cases}
$$

and note that by the Arzelà-Ascoli theorem, the sequence $\left\{\mu_{\ell}^{(k, w)}\right\}_{\ell \in \mathbb{N}} \subseteq$ $\mathrm{C}\left(\left[T_{k}, T_{k+1}\right] ; \mathbb{M}\right)$ contains a uniformly convergent subsequence. Taking sub-subsequences for $k \in[0: n]$ and $w \in \mathbb{W}^{n}$, we obtain a subsequence $\left\{\ell_{\nu}\right\}_{v \in \mathbb{N}}$ such that $\left\|\mu_{\ell_{v}}-\mu\right\|_{\text {sup }} \rightarrow 0$ as $v \rightarrow \infty$ for some $\mu \in \mathbb{D}(\mathbb{M})$. It is easy to see that $\mu \in \operatorname{Lip}(\mathbb{M})$, and thus Lip $(\mathbb{M})$ is indeed compact. Now Schauder's fixed point theorem implies that the continuous map $\chi: \operatorname{Lip}(\mathbb{M}) \rightarrow \operatorname{Lip}(\mathbb{M})$ has a fixed point $\mu \in \operatorname{Lip}(\mathbb{M})$; upon setting $v \triangleq \overleftarrow{\chi}[\mu] \in \operatorname{Reg}\left(\mathbb{R}^{d}\right)$ it follows that $\mu=\vec{\chi}[v] \in \operatorname{Reg}(\mathbb{M})$ and that $(\mu, v)$ is a solution of (E1)-(E6). 


\section{B Appendix: Proof of Theorem 16}

Proof of Theorem 16 Suppose that $\left(\mu_{1}, v_{1}\right)$ and $\left(\mu_{2}, v_{2}\right)$ are solutions of (E1)-(E6). In the following, we omit arguments to simplify notation when there is no ambiguity; e.g., $\widehat{\mathcal{H}}\left(\mu_{1}, v_{2}\right)=\widehat{\mathcal{H}}\left(t, w, \mu_{1}(t, w), v_{2}(t, w)\right)$.

Step 1: Dynamics between Common Noise Times. Let $k \in[0: n]$. The product rule and (E1)-(E2) yield

$$
\begin{aligned}
& \frac{\mathrm{d}}{\mathrm{d} t}\left[\left(\mu_{1}(t, w)-\mu_{2}(t, w)\right) \cdot\left(v_{1}(t, w)-v_{2}(t, w)\right)\right]=\frac{\mathrm{d}}{\mathrm{d} t}\left[\left(\mu_{1}-\mu_{2}\right) \cdot\left(v_{1}-v_{2}\right)\right] \\
& =\left[\mu_{1} \cdot \widehat{Q}\left(\mu_{1}, v_{1}\right)-\mu_{2} \cdot \widehat{Q}\left(\mu_{2}, v_{2}\right)\right] \cdot\left(v_{1}-v_{2}\right)-\left(\mu_{1}-\mu_{2}\right) \cdot\left[\widehat{\mathcal{H}}\left(\mu_{1}, v_{1}\right)-\widehat{\mathcal{H}}\left(\mu_{2}, v_{2}\right)\right] \\
& =\mu_{2} \cdot\left[\widehat{\mathcal{H}}\left(\mu_{2}, v_{1}\right)-\widehat{\mathcal{H}}\left(\mu_{2}, v_{2}\right)-\widehat{Q}\left(\mu_{2}, v_{2}\right) \cdot\left(v_{1}-v_{2}\right)\right] \\
& \quad+\mu_{1} \cdot\left[\widehat{\mathcal{H}}\left(\mu_{1}, v_{2}\right)-\widehat{\mathcal{H}}\left(\mu_{1}, v_{1}\right)-\widehat{Q}\left(\mu_{1}, v_{1}\right) \cdot\left(v_{2}-v_{1}\right)\right] \\
& \quad+\mu_{1} \cdot\left[\widehat{\mathcal{H}}\left(\mu_{2}, v_{2}\right)-\widehat{\mathcal{H}}\left(\mu_{1}, v_{2}\right)\right]+\mu_{2} \cdot\left[\widehat{\mathcal{H}}\left(\mu_{1}, v_{1}\right)-\widehat{\mathcal{H}}\left(\mu_{2}, v_{1}\right)\right]
\end{aligned}
$$

and thus by Assumptions 14(ii)-(iii) we obtain

$$
\begin{aligned}
& \frac{\mathrm{d}}{\mathrm{d} t}\left[\left(\mu_{1}(t, w)-\mu_{2}(t, w)\right) \cdot\left(v_{1}(t, w)-v_{2}(t, w)\right)\right] \\
& \quad \geq \gamma \cdot\left\|\mu_{1}(t, w)-\mu_{2}(t, w)\right\|^{\alpha}, \quad(t, w) \in\left[T_{k}, T_{k+1}\right\rangle \times \mathbb{W}^{n} .
\end{aligned}
$$

Step 2: Boundary Conditions at Common Noise Times. Let $k \in[1: n]$ and $w \in \mathbb{W}^{n}$. For $j=1,2$ and $\bar{w}_{k} \in \mathbb{W}$ we briefly write

$$
\begin{aligned}
& \mu_{j} \triangleq \mu_{j}\left(T_{k},\left(w_{-k}, \bar{w}_{k}\right)\right) \text { and } \mu_{j}^{-} \triangleq \mu_{j}\left(T_{k}-, w\right)=\mu_{j}\left(T_{k}-,\left(w_{-k}, \bar{w}_{k}\right)\right), \\
& v_{j} \triangleq v_{j}\left(T_{k},\left(w_{-k}, \bar{w}_{k}\right)\right), J\left(\mu_{j}^{-}\right) \triangleq J\left(T_{k},\left(w_{-k}, \bar{w}_{k}\right), \mu_{j}^{-}\right) \\
& \quad \text { and } \kappa_{k}\left(\mu_{j}^{-}\right) \triangleq \kappa_{k}\left(\bar{w}_{k} \mid w_{1}, \ldots, w_{k-1}, \mu_{j}^{-}\right) .
\end{aligned}
$$

By (E3) we have for $j, \ell=1,2$

$$
\mu_{j} \cdot v_{\ell}=\mu_{j}^{-} \cdot v_{\ell}^{J \cdot\left(\mu_{j}^{-}\right)} \text {for all } \bar{w}_{k} \in \mathbb{W}
$$

where it is understood that $\mu_{j}, v_{\ell}, J\left(\mu_{j}^{-}\right), \kappa_{k}\left(\mu_{j}^{-}\right)$depend on $\bar{w}_{k}$. Using (E4) in the first identity and (44) in the second and fourth, we find by elementary algebraic manipulations

$$
\begin{aligned}
& \left(\mu_{1}\left(T_{k}-, w\right)-\mu_{2}\left(T_{k}-, w\right)\right) \cdot\left(v_{1}\left(T_{k}-, w\right)-v_{2}\left(T_{k}-, w\right)\right) \\
& =\sum_{\bar{w}_{k} \in \mathbb{W}} \kappa_{k}\left(\mu_{1}^{-}\right) \cdot\left(\mu_{1}^{-}-\mu_{2}^{-}\right) \cdot v_{1}^{J \cdot\left(\mu_{1}^{-}\right)}-\sum_{\bar{w}_{k} \in \mathbb{W}} \kappa_{k}\left(\mu_{2}^{-}\right) \cdot\left(\mu_{1}^{-}-\mu_{2}^{-}\right) \cdot v_{2}^{J \cdot\left(\mu_{2}^{-}\right)} \\
& =\frac{1}{2} \sum_{\bar{w}_{k} \in \mathbb{W}} \kappa_{k}\left(\mu_{1}^{-}\right) \cdot\left(\mu_{1}^{-}-\mu_{2}^{-}\right) \cdot v_{1}^{J \cdot\left(\mu_{1}^{-}\right)} \\
& \quad+\frac{1}{2} \sum_{\bar{w}_{k} \in \mathbb{W}} \kappa_{k}\left(\mu_{1}^{-}\right) \cdot\left\{\left(\mu_{1}-\mu_{2}\right) \cdot v_{1}-\mu_{2}^{-} \cdot\left[v_{1}^{J \cdot\left(\mu_{1}^{-}\right)}-v_{1}^{J \cdot\left(\mu_{2}^{-}\right)}\right]\right\}
\end{aligned}
$$




$$
\begin{aligned}
& -\frac{1}{2} \sum_{\bar{w}_{k} \in \mathbb{W}} \kappa_{k}\left(\mu_{2}^{-}\right) \cdot\left(\mu_{1}^{-}-\mu_{2}^{-}\right) \cdot v_{2}^{J \cdot\left(\mu_{2}^{-}\right)} \\
& -\frac{1}{2} \sum_{\bar{w}_{k} \in \mathbb{W}} \kappa_{k}\left(\mu_{2}^{-}\right) \cdot\left\{\left(\mu_{1}-\mu_{2}\right) \cdot v_{2}+\mu_{1}^{-} \cdot\left[v_{2}^{J \cdot\left(\mu_{2}^{-}\right)}-v_{2}^{J \cdot\left(\mu_{1}^{-}\right)}\right]\right\} \\
= & \frac{1}{2} \sum_{\bar{w}_{k} \in \mathbb{W}}\left[\kappa_{k}\left(\mu_{1}^{-}\right)+\kappa_{k}\left(\mu_{2}^{-}\right)\right] \cdot\left(\mu_{1}-\mu_{2}\right) \cdot\left(v_{1}-v_{2}\right) \\
& -\frac{1}{2} \sum_{\bar{w}_{k} \in \mathbb{W}}\left[\kappa_{k}\left(\mu_{2}^{-}\right) \cdot v_{1}-\kappa_{k}\left(\mu_{1}^{-}\right) \cdot v_{2}\right] \cdot\left(\mu_{1}-\mu_{2}\right) \\
& +\frac{1}{2} \sum_{\bar{w}_{k} \in \mathbb{W}} \kappa_{k}\left(\mu_{1}^{-}\right) \cdot\left\{\left(\mu_{1}^{-}-\mu_{2}^{-}\right) \cdot v_{1}^{J \cdot\left(\mu_{1}^{-}\right)}-\mu_{2}^{-} \cdot\left[v_{1}^{J \cdot\left(\mu_{1}^{-}\right)}-v_{1}^{J \cdot\left(\mu_{2}^{-}\right)}\right]\right\} \\
& -\frac{1}{2} \sum_{\bar{w}_{k} \in \mathbb{W}} \kappa_{k}\left(\mu_{2}^{-}\right) \cdot\left\{\left(\mu_{1}^{-}-\mu_{2}^{-}\right) \cdot v_{2}^{J \cdot\left(\mu_{2}^{-}\right)}+\mu_{1}^{-} \cdot\left[v_{2}^{J \cdot\left(\mu_{2}^{-}\right)}-v_{2}^{J \cdot\left(\mu_{1}^{-}\right)}\right]\right\} \\
= & \frac{1}{2} \sum_{\bar{w}_{k} \in \mathbb{W}}\left[\kappa_{k}\left(\mu_{1}^{-}\right)+\kappa_{k}\left(\mu_{2}^{-}\right)\right] \cdot\left(\mu_{1}-\mu_{2}\right) \cdot\left(v_{1}-v_{2}\right) \\
& -\frac{1}{2} \sum_{\bar{w}_{k} \in \mathbb{W}}\left\{\kappa_{k}\left(\mu_{1}^{-}\right) \cdot\left[\mu_{2}^{-} \cdot v_{2}^{J \cdot\left(\mu_{2}^{-}\right)}-\mu_{1}^{-} \cdot v_{2}^{J \cdot\left(\mu_{1}^{-}\right)}\right]+\kappa_{k}\left(\mu_{2}^{-}\right) \cdot\left[\mu_{1}^{-} \cdot v_{1}^{J \cdot\left(\mu_{1}^{-}\right)}-\mu_{2}^{-} \cdot v_{1}^{J \cdot\left(\mu_{2}^{-}\right)}\right]\right\} \\
& +\frac{1}{2} \sum_{\bar{w}_{k} \in \mathbb{W}} \kappa_{k}\left(\mu_{1}^{-}\right) \cdot\left\{\mu_{1}^{-} \cdot v_{1}^{J \cdot\left(\mu_{1}^{-}\right)}-\mu_{2}^{-} \cdot v_{1}^{J \cdot\left(\mu_{2}^{-}\right)}-2 \mu_{2}^{-} \cdot\left[v_{1}^{J \cdot\left(\mu_{1}^{-}\right)}-v_{1}^{J \cdot\left(\mu_{2}^{-}\right)}\right]\right\} \\
& -\frac{1}{2} \sum_{\bar{w}_{k} \in \mathbb{W}} \kappa_{k}\left(\mu_{2}^{-}\right) \cdot\left\{2 \mu_{1}^{-} \cdot\left[v_{2}^{J \cdot\left(\mu_{2}^{-}\right)}-v_{2}^{J \cdot\left(\mu_{1}^{-}\right)}\right]+\mu_{1}^{-} \cdot v_{2}^{J \cdot\left(\mu_{1}^{-}\right)}-\mu_{2}^{-} \cdot v_{2}^{J \cdot\left(\mu_{2}^{-}\right)}\right\} \\
= & \frac{1}{2} \sum_{\bar{w}_{k} \in \mathbb{W}}\left[\kappa_{k}\left(\mu_{1}^{-}\right)+\kappa_{k}\left(\mu_{2}^{-}\right)\right] \cdot\left(\mu_{1}-\mu_{2}\right) \cdot\left(v_{1}-v_{2}\right) \\
& -\frac{1}{2} \sum_{\bar{w}_{k} \in \mathbb{W}}\left[\kappa_{k}\left(\mu_{1}^{-}\right)-\kappa_{k}\left(\mu_{2}^{-}\right)\right] \cdot\left[\mu_{2}^{-} \cdot v_{1}^{J \cdot\left(\mu_{2}^{-}\right)}-\mu_{1}^{-} \cdot v_{1}^{J \cdot\left(\mu_{1}^{-}\right)}+\mu_{2}^{-} \cdot v_{2}^{J \cdot\left(\mu_{2}^{-}\right)}-\mu_{1}^{-} \cdot v_{2}^{J \cdot\left(\mu_{1}^{-}\right)}\right] \\
& \left.-\kappa_{k}\left(\mu_{2}^{-}\right) \cdot \mu_{1}^{-} \cdot\left[v_{2}^{J \cdot\left(\mu_{2}^{-}\right)}-v_{2}^{J \cdot\left(\mu_{1}^{-}\right)}\right]+\kappa_{k}\left(\mu_{1}^{-}\right) \cdot \mu_{2}^{-} \cdot\left[v_{1}^{J \cdot\left(\mu_{1}^{-}\right)}-v_{1}^{J \cdot\left(\mu_{2}^{-}\right)}\right]\right\} . \\
& \\
& \\
&
\end{aligned}
$$

Hence by Assumption 14(iv) we conclude that

$$
\begin{aligned}
& \left(\mu_{1}^{-}-\mu_{2}^{-}\right) \cdot\left(v_{1}^{-}-v_{2}^{-}\right) \leq \frac{1}{2} \sum_{\bar{w}_{k} \in \mathbb{W}}\left[\kappa_{k}\left(\mu_{1}^{-}\right)+\kappa_{k}\left(\mu_{2}^{-}\right)\right] \cdot\left(\mu_{1}-\mu_{2}\right) \cdot\left(v_{1}-v_{2}\right) \\
& \quad \text { provided that }\left(\mu_{1}-\mu_{2}\right) \cdot\left(v_{1}-v_{2}\right)=\left[\Phi_{k}\left(w, \mu_{1}^{-}\right)-\Phi_{k}\left(w, \mu_{2}^{-}\right)\right] \cdot\left(v_{1}-v_{2}\right) \leq 0 .
\end{aligned}
$$

Step 3: Backward Propagation of Monotonicity. Suppose that for some $k \in[1: n]$ we have

$$
\left(\mu_{1}\left(T_{k+1}-, w\right)-\mu_{2}\left(T_{k+1}-, w\right)\right) \cdot\left(v_{1}\left(T_{k+1}-, w\right)-v_{2}\left(T_{k+1}-, w\right)\right) \leq 0 \text { for all } w \in \mathbb{W}^{n}
$$

By regularity, the fundamental theorem of calculus applies and using (43) we obtain

$$
\begin{aligned}
& \left(\mu_{1}\left(T_{k}, w\right)-\mu_{2}\left(T_{k}, w\right)\right) \cdot\left(v_{1}\left(T_{k}, w\right)-v_{2}\left(T_{k}, w\right)\right) \\
& =\left(\mu_{1}\left(T_{k+1}-, w\right)-\mu_{2}\left(T_{k+1}-, w\right)\right) \cdot\left(v_{1}\left(T_{k+1}-, w\right)-v_{2}\left(T_{k+1}-, w\right)\right)
\end{aligned}
$$


$-\int_{T_{k}}^{T_{k+1}} \frac{\mathrm{d}}{\mathrm{d} t}\left[\left(\mu_{1}(s, w)-\mu_{2}(s, w)\right) \cdot\left(v_{1}(s, w)-v_{2}(s, w)\right)\right] \mathrm{d} s \leq 0$ for all $w \in \mathbb{W}^{n}$

Thus (45) implies that (46) also holds at time $T_{k}-$, i.e.

$$
\left(\mu_{1}\left(T_{k}-, w\right)-\mu_{2}\left(T_{k}-, w\right)\right) \cdot\left(v_{1}\left(T_{k}-, w\right)-v_{2}\left(T_{k}-, w\right)\right) \leq 0 \text { for all } w \in \mathbb{W}^{n}
$$

Since (46) is satisfied for $k=n$ by Assumption 14(i), it follows by induction that

$$
\begin{aligned}
& \left(\mu_{1}\left(T_{k+1}-, w\right)-\mu_{2}\left(T_{k+1}-, w\right)\right) \cdot\left(v_{1}\left(T_{k+1}-, w\right)\right. \\
& \left.-v_{2}\left(T_{k+1}-, w\right)\right) \leq 0 \text { for all } k \in[0: n], w \in \mathbb{W}^{n}
\end{aligned}
$$

Step 4: Forward Propagation of Uniqueness. Suppose that for some $k \in[0: n]$ we have

$$
\mu_{1}\left(T_{k}, w\right)=\mu_{2}\left(T_{k}, w\right) \text { for all } w \in \mathbb{W}^{n}
$$

Using the fundamental theorem of calculus, (43) and (47) we find that

$$
\begin{aligned}
\gamma & \cdot \int_{T_{k}}^{T_{k+1}}\left\|\mu_{1}(s, w)-\mu_{2}(s, w)\right\|^{\alpha} \mathrm{d} s \\
\leq & \int_{T_{k}}^{T_{k+1}}\left(\frac{\mathrm{d}}{\mathrm{d} t}\left[\left(\mu_{1}(s, w)-\mu_{2}(s, w)\right) \cdot\left(v_{1}(s, w)-v_{2}(s, w)\right)\right]\right) \mathrm{d} s \\
= & \left(\mu_{1}\left(T_{k+1}-, w\right)-\mu_{2}\left(T_{k+1}-, w\right)\right) \cdot\left(v_{1}\left(T_{k+1}-, w\right)-v_{2}\left(T_{k+1}-, w\right)\right) \\
& \quad-\left(\mu_{1}\left(T_{k}, w\right)-\mu_{2}\left(T_{k}, w\right)\right) \cdot\left(v_{1}\left(T_{k+1}-, w\right)-v_{2}\left(T_{k+1}-, w\right)\right) \leq 0 \text { for all } w \in \mathbb{W}^{n}
\end{aligned}
$$

As a consequence, we have

$$
\mu_{1}(t, w)=\mu_{2}(t, w) \text { for all }(t, w) \in\left[T_{k}, T_{k+1}\right\rangle \times \mathbb{W}^{n}
$$

and, in particular, $\mu_{1}\left(T_{k+1}-, w\right)=\mu_{2}\left(T_{k+1}-, w\right)$, implying $\mu_{1}\left(T_{k+1}, w\right)=$ $\mu_{2}\left(T_{k+1}, w\right)$ for all $w \in \mathbb{W}^{n}$. Since $\mu_{1}(0)=m_{0}=\mu_{2}(0)$ by (E5), (48) is satisfied for $k=0$, and we conclude that $\mu_{1}=\mu_{2}$. Finally, since Assumption 14 subsumes Assumptions 11(i)-(ii), the arguments in the proof of Lemma A.2 yield $v_{1}=v_{2}$, and the proof is complete.

\section{References}

1. Ahuja, S.: Wellposedness of mean field games with common noise under a weak monotonicity condition. SIAM J. Control Optim. 54(1), 30-48 (2016)

2. Bayraktar, E., Cohen, A.: Analysis of a finite state many player game using its master equation. SIAM J. Control Optim. 56(5), 3538-3568 (2018)

3. Bayraktar, E., Cecchin, A., Cohen, A., Delarue, F.: Finite state mean field games with Wright-Fisher common noise (2019). arXiv:1912.06701

4. Bensoussan, A., Frehse, J., Yam, P.: Mean Field Games and Mean Field Type Control Theory. Springer Briefs in Mathematics. Springer, Berlin (2013) 
5. Bertucci, C., Lasry, J.-M., Lions, P.-L.: Some remarks on mean field games. Commun. Partial Differ. Equ. 44(3), 205-227 (2019)

6. Basei, M., Pham, H.: A weak martingale approach to linear-quadratic McKean-Vlasov stochastic control problems. J. Optim. Theory Appl. 181(2), 347-382 (2019)

7. Becherer, D., Schweizer, M.: Classical solutions to reaction-diffusion systems for hedging problems with interacting Itô and point processes. Ann. Appl. Probab. 15(2), 1111-1144 (2005)

8. Carmona, R., Delarue, F.: Probabilistic analysis of mean-field games. SIAM J. Control Optim. 51(4), 2705-2734 (2013)

9. Carmona, R., Delarue, F.: Probabilistic Theory of Mean Field Games with Applications I: Mean Field FBSDEs, Control, and Games. Springer, Berlin (2018a)

10. Carmona, R., Delarue, F.: Probabilistic Theory of Mean Field Games with Applications II: Mean Field Games with Common Noise and Master Equations. Springer, Berlin (2018)

11. Carmona, R., Delarue, F., Lachapelle, A.: Control of McKean-Vlasov dynamics versus mean field games. Math. Financ. Econ. 7(2), 131-166 (2013)

12. Carmona, R., Delarue, F., Lacker, D.: Mean field games with common noise. Ann. Probab. 44(6), 3740-3803 (2016)

13. Carmona, R., Delarue, F., Lacker, D.: Mean field games of timing and models for bank runs. Appl. Math. Optim. 76(1), 217-260 (2017)

14. Campi, L., Fischer, M.: $N$-player games and mean-field games with absorption. Ann. Appl. Probab. 28(4), 2188-2242 (2018)

15. Cecchin, A., Fischer, M.: Probabilistic approach to finite state mean field games. Appl. Math. Optim. 81(2), 253-300 (2020)

16. Carmona, R., Fouque, J.-P., Sun, L.-H.: Mean field games and systemic risk. Commun. Math. Sci. 13(4), 911-933 (2015)

17. Casgrain, P., Jaimungal, S.: Mean field games with partial information for algorithmic trading (2019). arxiv: 1803.04094

18. Cecchin, A., Pelino, G.: Convergence, fluctuations and large deviations for finite state mean field games via the master equation. Stoch. Process. Appl. 129(11), 4510-4555 (2019)

19. Cosso, A., Pham, H.: Zero-sum stochastic differential games of generalized McKean-Vlasov type. J. Math. Pures Appl. 129, 180-212 (2019)

20. Cecchin, A., Dai Pra, P., Fischer, M., Pelino, G.: On the convergence problem in mean field games: a two state model without uniqueness. SIAM J. Control Optim. 57(4), 2443-2466 (2019)

21. Carmona, R., Wang, P.: An alternative approach to mean field game with major and minor players, and applications to herders impacts. Appl. Math. Optim. 76(1), 5-27 (2017)

22. Carmona, R., Wang, P.: A probabilistic approach to extended finite state mean field games (2018). arXiv:1808.07635

23. Doncel, J., Gast, N., Gaujal, B.: A mean-field game analysis of SIR dynamics with vaccination (2017). hal:01496885

24. Doncel, J., Gast, N., Gaujal, B.: Discrete mean field games: existence of equilibria and convergence. J. Dyn. Games 6(3), 221-239 (2019)

25. Delarue, F., Lacker, D., Ramanan, K.: From the master equation to mean field game limit theory: a central limit theorem. Electron. J. Probab. 24(51), 54 (2019)

26. Elie, R., Hubert, E., Turinici, G.: Contact rate epidemic control of COVID-19: an equilibrium view (2020). arxiv:2004.08221

27. Elie, R., Ichiba, T., Laurière, M.: Large banking systems with default and recovery: a mean field game model (2020). arxiv:2001.10206

28. Fischer, M.: On the connection between symmetric $n$-player games and mean field games. Ann. Appl. Probab. 27(2), 757-810 (2017)

29. Guéant, O., Lasry, J.-M., Lions, P.-L.: Mean field games and applications. In: Carmona, R., Çınlar, E., Ekeland, I., Jouini, E., Scheinkman, J.A., Touzi, N. (eds.) Paris-Princeton Lectures on Mathematical Finance 2010. Lecture Notes in Mathematics, pp. 205-266. Springer, New York (2011)

30. Gomes, D.A., Mohr, J., Souza, R.R.: Discrete time, finite state space mean field games. J. Math. Pures Appl. 93(3), 308-328 (2010)

31. Gomes, D.A., Mohr, J., Souza, R.R.: Continuous time finite state mean field games. Appl. Math. Optim. 68(1), 99-143 (2013) 
32. Grimm, V., Mengel, F., Schmidt, M.: Extensions of the SEIR model for the analysis of tailored social distancing and tracing approaches to cope with COVID-19. medRxiv (2020). https://www.medrxiv. org/content/10.1101/2020.04.24.20078113v1

33. Gomes, D.A., Saúde, J.: A mean-field game approach to price formation. Dyn. Games Appl. (to appear, 2020)

34. Guéant, O.: Existence and uniqueness result for mean field games with congestion effect on graphs. Appl. Math. Optim. 72(2), 291-303 (2015)

35. Gomes, D., Velho, R.M., Wolfram, M.-T.: Socio-economic applications of finite state mean field games. Philosoph. Trans. R. Soc. A 372, 2014 (2028)

36. Hale, J.K.: Ordinary Differential Equations. Robert E. Krieger Publishing Company, Inc., New York (1980)

37. Hethcote, H.W.: The mathematics of infectious diseases. SIAM Rev. 42(4), 599-653 (2000)

38. Harko, T., Lobo, F.S.N., Mak, M.K.: Exact analytical solutions of the susceptible-infected-recovered (SIR) epidemic model and of the SIR model with equal death and birth rates. Appl. Math. Comput. 236, 184-194 (2014)

39. Huang, M., Malhamé, R.P., Caines, P.E.: Large population stochastic dynamic games: Closed-loop McKean-Vlasov systems and the Nash certainty equivalence principle. Commun. Inform. Syst. 6(3), 221-252 (2006)

40. Kolokoltsov, V.N., Bensoussan, A.: Mean-field-game model for botnet defense in cyber-security. Appl. Math. Optim. 74(3), 669-692 (2016)

41. Kolokoltsov, V.N., Malafeyev, O.A.: Mean-field-game model of corruption. Dyn. Games Appl. 7(1), 34-47 (2017)

42. Lacker, D.: A general characterization of the mean field limit for stochastic differential games. Probab. Theory Relat. Fields 165(3-4), 581-648 (2015)

43. Lacker, D.: On a strong form of propagation of chaos for McKean-Vlasov equations. Electron. Commun. Probab. 23(45), 11 (2018)

44. Lasry, J.-M., Lions, P.-L.: Mean field games. Jpn. J. Math. 2(1), 229-260 (2007)

45. Lachapelle, A., Lasry, J.-M., Lehalle, C.-A., Lions, P.-L.: Efficiency of the price formation process in presence of high frequency participants: a mean field game analysis. Math. Financ. Econ. 10(3), 223-262 (2016)

46. Laguzet, L., Turinici, G.: Individual vaccination as Nash equilibrium in a SIR model with application to the 2009-2010 Influenza A (H1N1) epidemic in France. Bull. Math. Biol. 77(10), 1955-1984 (2015)

47. Miller, J.C.: Mathematical models of SIR disease spread with combined non-sexual and sexual transmission routes. Infect. Dis. Model. 2(1), 35-55 (2017)

48. Miller, E., Pham, H.: Linear-quadratic McKean-Vlasov Stochastic differential games. In: Yin, G., Zhang, Q. (eds.) Modeling, Stochastic Control, Optimization, and Applications (The IMA Volumes in Mathematics and its Applications), vol. 164, pp. 451-481. Springer, New York (2019)

49. Neumann, B.A.: Stationary equilibria of mean field games with finite state and action space. Dyn. Games Appl. (to appear, 2020)

50. Nutz, M.: A mean field game of optimal stopping. SIAM J. Control Optim. 56(2), 1206-1221 (2018)

51. Pham, H., Wei, X.: Dynamic programming for optimal control of stochastic McKean-Vlasov dynamics. SIAM J. Control Optim. 55(2), 1069-1101 (2017)

52. Wang, P.: Finite state mean field games. Dissertation, Princeton University, Princeton, NJ (2019). http:// arks.princeton.edu/ark:/88435/dsp01zw12z808g

Publisher's Note Springer Nature remains neutral with regard to jurisdictional claims in published maps and institutional affiliations. 